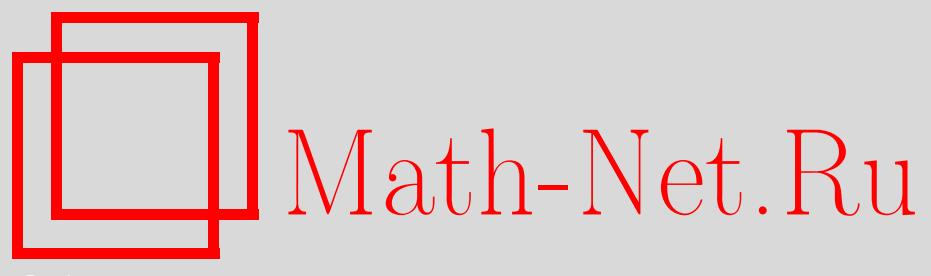

Л. А. Бекларян, Группы гомеоморфизмов прямой и окружности. Метрические инварианты и вопросы классификации, УМН, 2015, том 70, выпуск 2, 3-54

DOI: https://doi.org/10.4213/rm9654

Использование Общероссийского математического портала Math-Net.Ru подразумевает, что вы прочитали и согласны с пользовательским соглашением http://www . mathnet.ru/rus/agreement

Параметры загрузки:

IP : 3.95 .254 .165

26 апреля 2023 г., 10:21:58

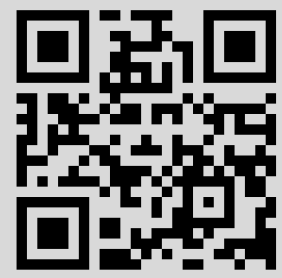




\section{Группы гомеоморфизмов прямой и окружности. Метрические инварианты и вопросы классификации}

\section{Л. А. Бекларян}

В обзоре обсуждается подход для классификации групп гомеоморфизмов прямой и окружности, основанный на цепочках вложений и соответствиях классов групп, порожденных различными характеристиками групп.

Библиография: 60 названий.

Ключевые слова: группы гомеоморфизмов прямой (окружности), проективно инвариантные меры, аменабельность, свободные подгруппы, рост группы.

DOI: $10.4213 / \mathrm{rm} 9654$

\section{СоДЕРЖАНИЕ}

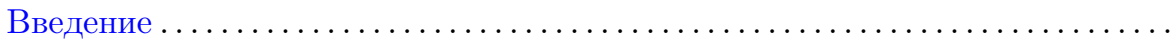

1. Аменабельность и парадоксальные разбиения. Число Тарского и про-

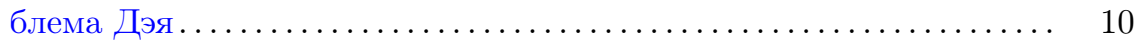

2. Рост конечно порожденной группы и соответствия классов групп...... 15

3. О реализациях абстрактных групп как групп действий на прямой (ок-

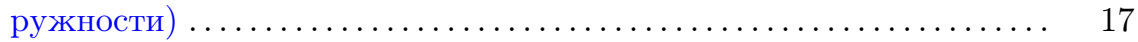

4. Топологические характеристики и метрические инварианты групп го-

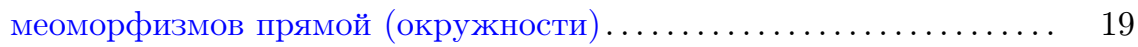

4.1. Минимальные множества ............................. 20

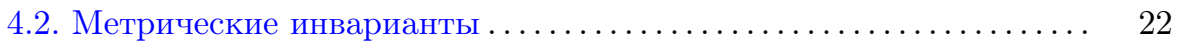

5. Цепочки вложений групп гомеоморфизмов прямой (окружности, интервала) и факторгрупп, а также соответствия классов таких групп и факторгрупп, порожденные различными их характеристиками .

5.1. Цепочки вложений факторгрупп и соответствия классов групп гомеоморфизмов прямой (окружности), порожденные метриче-

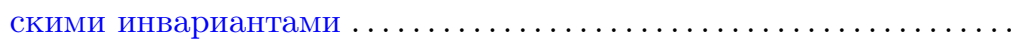

5.2. Цепочки вложений групп гомеоморфизмов прямой (окружности, интервала) и факторгрупп, а также соответствия классов таких групп и факторгрупп, порожденные их ростом ............

Работа выполнена при поддержке РФФИ (грант № 12-01-00768-а) и программы "Ведущие научные школы" (грант НШ-5998.2012.1).

(C) Л.А. БЕклАРян, 2015 
6. Схема классификации групп гомеоморфизмов прямой (окружности, ин-

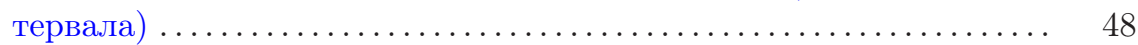

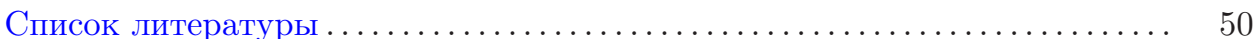

\section{Введение}

Работа посвящена классификации групп гомеоморфизмов прямой и окружности, основанной на цепочках вложений и соответствиях классов групп, порожденных различными характеристиками групп. Группы гомеоморфизмов прямой (окружности) возникают при изучении различных задач геометрии, групп квазиконформных отображений, функционально-дифференциальных уравнений, теории волн, вариационного исчисления и т.д. [3], [4], [12], [15], [42], [54]. Изучение подобных групп основано на методах и подходах, накопленных в теории абстрактных групп. K этим методам относится классификация групп, основанная на наличии у групп различных характеристик. Такими характеристиками могут служить число Тарского, свойство аменабельности, рост группы (для конечно порожденных групп), существование (отсутствие) свободной подполугруппы (подгруппы) с двумя образующими, структура орбит (для групп гомеоморфизмов локально компактного пространства) и т. д. Например, число Тарского может быть основой для формирования соответствий классов групп только лишь внутри подмножества парадоксальных (неаменабельных) групп, а рост группы - только лишь внутри подмножества конечно порожденных аменабельных групп. Другим способом является изучение цепочек вложений различных классов групп, например цепочки вложений для классов групп трех типов в множестве всех групп: элементарных групп; аменабельных групп; групп, не содержащих свободных подгрупп с двумя образующими. Такая задача известна как проблема Дэя. Множество всех групп имеет весьма сложную структуру, поэтому трудно ожидать у таких групп существование тех или иных характеристик, связанных со структурой группы и позволяющих провести достаточно детальную классификацию. Тем не менее для некоторого подмножества множества всех групп такая классификация может оказаться более информативной. В роли такого подмножества групп в нашем случае рассматриваются группы гомеоморфизмов прямой и окружности. Вместе с тем следует отметить, что группы гомеоморфизмов прямой (окружности) сами являются представительным подмножеством в множестве всех абстрактных групп, ибо всякая правоупорядочиваемая счетная группа может быть реализована как группа гомеоморфизмов прямой. Такие группы имеют весьма сложную структуру и, в частности, содержат группы промежуточного роста.

Особенность групп гомеоморфизмов прямой и окружности состоит в том, что для них существует ряд метрических инвариантов, а именно инвариантная мера, проективно инвариантная мера, $\omega$-проективно инвариантная мера. Наличие метрических инвариантов позволяет для групп гомеоморфизмов прямой и окружности провести достаточно детальную классификацию, основанную на характеристиках этих метрических инвариантов [8]-[11]. K наиболее 
информативным характеристикам относятся те, которые формируют критерии существования таких метрических инвариантов. Характеристики могут быть сформулированы в различных терминах исходной группы: топологических характеристик, канонических подгрупп, комбинаторных характеристик, характеристик структуры орбит и т. д. Очевидно, что чем больше разнообразных переформулировок критериев существования метрических инвариантов, тем больше разнообразных соответствий классов групп, основанных на них.

В разделе 1 обсуждается число Тарского [57] и связанные с ним соответствия классов абстрактных групп. Там же представлена проблема Дэя [23] как пример задания цепочки точных вложений. Точность рассматриваемой цепочки вложений изучалась в работах Адяна [2], Ольшанского [43], Громова [34], Григорчука [30]. Соответствия классов абстрактных групп, порожденные числом Тарского, являются информативными только лишь внутри подмножества парадоксальных групп. Обсуждаемая же цепочка точных вложений информативна как внутри подмножества аменабельных групп, так и внутри подмножества парадоксальных групп, пограничных (в смысле числа Тарского) как с аменабельными группами, так и с группами, содержащими свободные подгруппы с двумя образующими.

В разделе 2 представлены как уточненная цепочка точных вложений для подмножества конечно порожденных абстрактных групп, так и соответствия классов абстрактных групп, порожденные ростом группы. Отмечено, что разбиение на классы групп (соответствия классов групп), порожденное ростом группы, обладает рядом недостатков. Например, экспоненциальным ростом может обладать как разрешимая группа (такая группа принадлежит подмножеству элементарных групп), так и группа со свободной подгруппой с двумя образующими. Следовательно, в подмножестве конечно порожденных абстрактных групп нет достаточно детализированных соответствий классов групп, порожденных ростом группы. Вместе с тем в множестве абстрактных групп для класса почти нильпотентных групп существует однозначное соответствие с их полиномиальным ростом, даваемое теоремой Громова [35]. Для конечно порожденных разрешимых групп приводится критерий почти нильпотентности группы, установленный Розенблаттом [48]. Отмечено, что в подмножестве конечно порожденных абстрактных групп существуют не только группы полиномиального и экспоненциального роста, но и группы промежуточного роста, что и было установлено Григорчуком [30].

Раздел 3 посвящен обсуждению вопроса о месте подмножества групп гомеоморфизмов прямой, окружности, интервала в множестве всех абстрактных групп. С одной стороны, такие группы являются группами действий и, соответственно, обладают рядом дополнительных характеристик по сравнению с абстрактными группами. С другой стороны, ряд абстрактных групп допускает реализацию в виде групп гомеоморфизмов прямой. Такие конструкции представлены в работах Григорчука [33], Гиза [28]. В частности, в [33] показано, что группа, ассоцированная с группой Григорчука, может быть реализована как группа гомеоморфизмов интервала (прямой) и, соответственно, является группой промежуточного роста. Этот факт указывает на то, что группа всех 
гомеоморфизмов интервала (прямой) имеет сложную структуру. Оказывается, ряд абстрактных групп также можно реализовать не только как группы гомеоморфизмов прямой, окружности, интервала, но и как группы диффеоморфизмов. Эти вопросы исследовали Фарб и Фрэнкс [27], Навас, Деруан и Клепцын [25]. В частности, Навас [25] показал, что группа, ассоциированная с группой Григорчука, может быть реализована и как группа $C^{1}$-диффеоморфизмов интервала и, соответственно, также является группой промежуточного роста. Особое место занимают нильпотентные группы (без кручения). Фарб и Фрэнкс [27] показали, что такие группы могут быть реализованы как группы $C^{1}$-диффеоморфизмов интервала, а Планте и Тёрстон [46], Деруан и Клепцын [25] показали, что класс гладкости диффеоморфизма накладывает ограничения на структуру группы (ступень нильпотентности).

Раздел 4 посвящен обсуждению топологических характеристик и метрических инвариантов группы гомеоморфизмов прямой и окружности. Все свойства такой группы основаны на существовании некоторого естественного частичного порядка на такой группе и справедливости теоремы Гёльдера об архимедовых группах для факторгруппы по минимальной подгруппе, содержащей множество всех стабилизаторов. Этот факт отражен в теореме о факторгруппе, играющей ключевую роль в исследовании групп гомеоморфизмов прямой и окружности. Обсуждается вопрос о классификационных возможностях теоремы о факторгруппе.

Для произвольной группы гомеоморфизмов прямой (окружности) теорема о факторгруппе была доказана в работе автора [6]. Для специальных групп гомеоморфизмов прямой, сохраняющих ориентацию, утверждение теоремы о факторгруппе было получено в работах Солодова [53], Новикова [42], Сали [49]-[51]. Новиков [42] изучал свободно действующие группы, Солодов [53] - конечно порожденные группы, не содержащие свободных подполугрупп с двумя образующими, а Сали [49]-[51] - счетные группы с общей неподвижной точкой для элементов, являющихся стабилизаторами.

Пункт 4.1 посвящен минимальным множествам. Важность минимальных множеств состоит в том, что они определяют структуру орбит, а также носители инвариантных мер, проективно инвариантных мер и их эргодические свойства. Последних отмеченных свойств минимальных множеств мы в данной работе касаться не будем. Более развернутое изложение всех этих вопросов имеется в [14]. Описана структура минимальных множеств. Показано, что их структура каноническая, и выделяются четыре типа: пустое множество; дискретное множество; множество, гомеоморфное канторову множеству; все пространство. Приводится признак непустоты минимального множества (в случае окружности минимальное множество всегда непусто), а также изучаются свойства наследования для минимальных множеств. Для произвольной группы гомеоморфизмов прямой и окружности минимальные множества изучались в работах автора [10], [11], [14]. Минимальные множества для счетных групп гомеоморфизмов прямой исследовались в работах Сали [49]-[51], в которых сформулированы признаки существования непустых минимальных множеств и при этих условиях описана структура орбит. Там же, а также в работе 
Планте [45] исследовались признаки существования непустых минимальных множеств для специальных групп диффеоморфизмов. Минимальные множества для произвольных групп гомеоморфизмов окружности описывались различными авторами, в частности в работах Сали [49]-[51] для счетных групп, в работе Карловича [36] для свободно действующих групп, и др. Наиболее ранней работой по этой теме является курс лекций Альфорса [4]. Во многих задачах, включая вопросы классификации, важную роль играет факт наличия (отсутствия) минимального множества, гомеоморфного канторову множеству. Для гомеоморфизма окружности первый результат об отсутствии минимального множества, изоморфного канторову множеству, в предположении гладкости был получен Данжуа [38] для циклической группы; этот результат известен под названием теоремы Данжуа. Очевидно, что справедливость теоремы Данжуа следует ожидать только для специальных групп.

Пункт 4.2 посвящен метрическим инвариантам. Обсуждается метрический инвариант в форме $\omega$-проективно инвариантной меры (определение которого было дано в работе автора [11]) и установлено существование такого инварианта для групп с непустым минимальным множеством. Оказывается, что такой метрический инвариант обладает следующим экстремальным свойством [14]: кардинальное число $\omega$ либо равно 1 , либо бесконечно. В случае $\omega=1$ такой инвариант переходит в хорошо известную проективно инвариантную меру, частным случаем которой является инвариантная мера. В связи с этим следует отметить, что для групп с проективно инвариантной мерой (инвариантной мерой) минимальное множество непусто.

Подпункт а) посвящен обсуждению инвариантной меры. K наиболее ранним результатам по инвариантным мерам относится теорема Боголюбова-Крылова-Дэя [37], [23] о существовании инвариантной меры для аменабельной группы действий на компакте (признак существования инвариантной меры). Тем не менее в течение длительного времени критерий существования инвариантной меры отсутствовал. Важное продвижение в этом вопросе принадлежит Планте [44], который для конечно порожденной группы гомеоморфизмов прямой (окружности) получил критерий существования инвариантной меры, сформулированный в терминах асимптотических свойств орбит. Важно, что это именно критерий и он справедлив даже в случае некомпактности пространства действий, т. е. в случае прямой. К сожалению, такой критерий не может быть распространен на произвольные группы гомеоморфизмов прямой (окружности). Точно так же в случае окружности (компактный случай) не удавалось соотнести условия теорем Планте и Боголюбова-Крылова-Дэя. Очевидно, что существование инвариантной меры эквивалентно условию непустоты его носителя. На этом пути в работе [10] автором и был сформулирован критерий существования инвариантной меры для произвольной группы гомеоморфизмов прямой (окружности). Удалось определить топологический инвариант в виде замкнутого инвариантного подмножества прямой (окружности), непустота которого эквивалентна непустоте носителя инвариантной меры. В свою очередь, условие непустоты такого замкнутого инвариантного подмножества прямой (окружности) удается сформулировать в различных терминах, что и позволило дать пе- 
реформулировки критерия существования инвариантной меры. Обсуждается ряд таких переформулировок, имеющих вид строгой альтернативы. В случае окружности одна из таких переформулировок является неулучшаемым усилением теоремы Боголюбова-Крылова-Дэя.

Подпункт b) посвящен обсуждению проективно инвариантной меры. Инвариантная мера является частным случаем более общего инварианта, а именно проективно инвариантной меры. Поэтому изучение проективно инвариантной меры становится актуальным, когда отсутствует какая-либо инвариантная мера. В работе автора [9] для групп гомеоморфизмов прямой без инвариантной меры в терминах выделенных подмножеств и геометрии взаиморасположения графиков гомеоморфизмов был сформулирован критерий существования проективно инвариантной меры. Далее, в [10] для групп гомеоморфизмов прямой без инвариантной меры удалось сформулировать критерий существования проективно инвариантной меры, основанный только лишь на свойствах выделенных подмножеств. Но описание самих выделенных подмножеств может оказаться сложной задачей. Чтобы обойти отмеченную сложность, был получен критерий существования проективно инвариантной меры, основанный на существовании нормальной подгруппы с некоторыми заданными свойствами. Среди свойств такой нормальной подгруппы - наличие свободно действующего элемента, существование инвариантной меры и наличие некоторой "алгебраической сложности" самой подгруппы. Важное замечание состоит в том, что для групп гомеоморфизмов прямой без инвариантной меры, но с проективно инвариантной мерой обязательно существует свободно действующий гомеоморфизм. В критерии проверка "алгебраической сложности" самой подгруппы также является весьма нетривиальной. В связи с этим актуален вопрос: можно ли в критерии это условие заменить условиями другого типа? Ответ на этот вопрос оказывается положительным. В [10] для групп гомеоморфизмов прямой, содержащих нормальную подгруппу с инвариантной мерой и свободно действующим элементом, получен критерий существования проективно инвариантной меры, основанный на комбинаторном свойстве в виде отсутствия свободной подгруппы с двумя образующими для факторгруппы по подгруппе стабилизаторов, оставляющих на месте все точки минимального множества. Достаточное условие справедливости такого утверждения, но при более сильном предположении об аменабельности исходной группы, было получено в работе Планте [45]. Вместе с тем условие существования нормальной подгруппы со свободно действующим элементом также является трудно проверяемым. Позднее в [19] для групп гомеоморфизмов прямой был получен критерий существования проективно инвариантной меры в терминах коммутанта. Там же для групп, содержащих свободно действующий элемент, на основе такого критерия удалось получить критерий существования проективно инвариантной меры, основанный на отсутствии подгруппы с двумя образующими, графики которых имеют заданную геометрию.

Опишем вкратце содержание раздела 5. С минимальным множеством связана важная факторизация исходной группы гомеоморфизмов прямой (окружности) по подгруппе стабилизаторов, оставляющих на месте все точки мини- 
мального множества. Обсуждаются цепочки вложений для таких факторгрупп и самих исходных групп, а также соответствия классов факторгрупп и групп, порожденные различными их характеристиками. $\mathrm{K}$ таким характеристикам относятся инвариантная мера, проективно инвариантная мера, $\omega$-проективно инвариантная мера, а также рост группы для конечно порожденных групп. Отдельные фрагменты рассматриваемых одноименных цепочек вложений для факторгрупп и групп почти очевидны. Важно то, что каждая из рассматриваемых характеристик порождает свое соответствие классов, в частности классов из рассматриваемой цепочки вложений. И чем больше характеристик с такими свойствами, тем больше возможностей для описания классов. Оказывается, что цепочки вложений рассматриваемых групп и факторгрупп, а также соответствия классов таких групп и факторгрупп, порожденные метрическими инвариантами, максимально информативны для описания классов именно факторгрупп, но не для классов исходных групп (см. п. 5.1). В силу этого препятствием к более детальному описанию групп гомеоморфизмов прямой (окружности) с непустым минимальным множеством является отсутствие описания структуры подгруппы стабилизаторов, оставляющих на месте все точки минимального множества. Подход, используемый для дальнейшего изучения этой подгруппы, и определяет процедуру индукции, на которой основана схема классификации групп гомеоморфизмов прямой, описанная в разделе 6. Для специальных групп диффеоморфизмов (см. п. 5.2) цепочки вложений самих исходных групп и соответствия классов таких групп также оказываются максимально информативными. Важной задачей остается описание структуры группы с пустым минимальным множеством.

Пункт 5.1 посвящен изучению цепочек вложений для факторгрупп, а также соответствий классов групп гомеоморфизмов прямой (окружности), порожденных метрическими инвариантами.

В подпункте а) сформулированная задача изучается в случае инвариантной меры. В частности, для класса групп, совпадающих со своей факторизацией, сформулирован критерий коммутативности. В подпункте b) сформулированная задача изучается в случае проективно инвариантной меры. В частности, для класса групп, совпадающих со своей факторизацией, сформулирован критерий разрешимости. Подпункт с) посвящен обсуждению соответствий классов групп, порожденных инвариантной, проективно инвариантной, а также $\omega$-проективно инвариантной мерами.

Пункт 5.2 посвящен изучению цепочек вложений конечно порожденных групп гомеоморфизмов прямой (окружности, интервала) и факторгрупп, а также соответствий классов таких групп и факторгрупп, порожденных их ростом.

В подпункте а) рассматриваются гомеоморфизмы прямой и окружности, сформулированная задача изучается для факторгрупп. Установлено, что рост таких факторгрупп имеет экстремальное свойство: он либо полиномиальный, либо экспоненциальный. Из такого свойства, в частности, следует, что группа промежуточного роста не может совпадать со своей факторизацией. В подпункте b) сформулированная задача изучается для конечно порожденных групп диффеоморфизмов интервала из $C^{(1+\alpha)}$ и групп диффеоморфизмов окружно- 
сти из $C^{2}$ с взаимно трансверсальными элементами. Цепочки вложений, а также соответствия классов групп первого и второго типа основаны на теоремах Наваса [41] и автора [17] соответственно, в которых устанавливается критерий почти нильпотентности. Из такого критерия, в частности, следует, что не существует групп первого и второго типа, имеющих промежуточный рост, т. е. рост таких групп также имеет экстремальное свойство: он либо полиномиальный, либо экспоненциальный. В действительности, для таких групп многие важные свойства являются следствием справедливости теоремы Данжуа об отсутствии минимальных множеств, изоморфных канторому множеству.

Раздел 6 посвящен обсуждению схемы классификации групп гомеоморфизмов прямой (окружности, интервала), основанной на свойствах цепочек вложений для факторгрупп.

\section{1. Аменабельность и парадоксальные разбиения. Число Тарского и проблема Дэя}

Всюду в статье все группы будут рассматриваться как дискретные группы. С понятием аменабельности связаны важнейшие характеристики группы, в частности инвариантные меры. Этот факт известен начиная с ранних работ Н. М. Крылова и Н.Н. Боголюбова по инвариантным мерам для групп, действующих на компакте. Приведем определение аменабельности в терминах метрического инварианта.

ОпредЕление 1. Дискретная группа $G$ называется аменабельной, если она допускает $G$-инвариантную вероятностную меру, т. е. отображение

$$
\mu: P(G) \rightarrow[0,1]
$$

$(P(G)$ - множество всех подмножеств $G)$, обладающее следующими свойствами:

1) конечная аддитивность;

2) $\mu(g A)=\mu(A)$ для всех $g \in G, A \subseteq G$;

3) $\mu(G)=1$.

Дадим эквивалентное функиионально-аналитическое определение аменабельности. Для группы $G$ через $B(G)$ обозначим пространство ограниченных функций на $G$ с sup-нормой.

Линейный функционал $m$ на $B(G)$ называется левоинвариантным средним, если:

1) $m(\bar{f})=\overline{m(f)}$

2) $m(f) \geqslant 0, f \geqslant 0, m(1)=1$;

3) $m(g f)=m(f)$, где $g f(\bar{g})=f\left(g^{-1} \bar{g}\right)$ для всех $g, \bar{g} \in G, f \in B(G)$.

ОпредЕлЕниЕ $1^{*}$. Дискретная группа $G$ называется аменабельной, если существует левоинвариантное среднее.

Аменабельность может быть определена и в терминах комбинаторных характеристик группы в виде условия Фельнера. 
ОпределЕниЕ $1^{* *}$. Дискретная группа $G$ называется аменабельной, если для любого $\varepsilon>0$ и любого конечного множества $I \subset G$ найдется непустое конечное множество $U \subset G$ такое, что $|U|^{-1}|g U \triangle U|<\varepsilon$ при всех $g \in I$, где $|U|$ - мощность $U$.

Для конечно порожденной группы $G=\left\langle g_{1}, \ldots, g_{s}\right\rangle$ подмножества $U_{k} \subset G$, $\left|U_{k}\right|<+\infty, k=1,2, \ldots$, называются фельнеровской последовательностью относительно множества образующих $\left\{g_{1}, \ldots, g_{s}\right\}$, если для любого $\varepsilon>0$ найдется $N>0$ такое, что для всех $k>N$ выполняются условия $\left|g_{j} U_{k} \triangle U_{k}\right|<\varepsilon$, $j=1, \ldots, s$. Для таких групп аменабельность эквивалентна существованию фельнеровской последовательности.

Свойство неаменабельности, как альтернативное к аменабельности, также допускает определение в терминах комбинаторных характеристик группы.

ОпредЕлЕниЕ 2. Группа $G$ парадоксальна, если $G$ допускает парадоксальное разбиение: существуют подмножества $A_{1}, \ldots, A_{n}, B_{1}, \ldots, B_{m}$ в $G$ и элементы $g_{1}, \ldots, g_{n}, h_{1}, \ldots, h_{m}$ такие, что

$$
G=A_{1} \sqcup \cdots \sqcup A_{n} \sqcup B_{1} \sqcup \cdots \sqcup B_{m}=g_{1} A_{1} \sqcup \cdots \sqcup g_{n} A_{n}=h_{1} B_{1} \sqcup \cdots \sqcup h_{m} B_{m} .
$$

Теорема 1 (альтернатива Тарского, 1949 г., [57]). Групnа G либо аменабельна, либо парадоксальна.

В частности, группа, содержащая свободную подгруппу с двумя образующими, является парадоксальной.

Множество всех аменабельных групп, обозначаемое через $\mathscr{A}$, замкнуто относительно следующих четырех операций:

(i) взятия подгруппы аменабельных групп;

(ii) взятия факторгруппы аменабельных групп;

(iii) расширения аменабельных групп с помощью аменабельных ( $G$ является расширением группы $H$ посредством $F$, если $H \unlhd G$ и $G / H \cong F)$; (iv) направленного объединения аменабельных подгрупп $\left\{H_{\alpha}\right\}\left(\bigcup_{\alpha} H_{\alpha}\right.$ и для
любых $H_{\alpha_{1}}, H_{\alpha_{2}}$ существует $\left.H_{\gamma} \supset H_{\alpha_{1}} \cup H_{\alpha_{2}}\right)$.

Из перечисленных свойств множества $A G$ следует ряд свойств для парадоксальных групп:

1) если подгруппа $H$ группы $G$ парадоксальна, то и $G$ парадоксальна;

2) если факторгруппа $H$ группы $G$ парадоксальна, то и $G$ парадоксальна.

Для парадоксальных групп определяется число Тарского.

ОПРЕДЕлЕНиЕ 3 . Если группа $G$ парадоксальна, то минимальное число $\tau=$ $n+m$ среди всех парадоксальных разбиений называется числом Тарского и обозначается через $\tau(G)$.

Из определения парадоксальности (определение 2) легко следует, что всегда справедлива оценка снизу

$$
\tau(G) \geqslant 4 .
$$

Для аменабельной группы $G$ по определению положим

$$
\tau(G)=+\infty .
$$


Приведем несколько утверждений относительно числа Тарского.

УтВЕРЖДЕНИЕ 1. Если подгруппа $H$ (факторгруппа) парадоксальна, то $\tau(G) \leqslant \tau(H)$.

Оказывается, что для каждой парадоксальной группы существует конечно порожденная подгруппа с тем же числом Тарского, т. е. сложность парадоксальной группы достигается на некоторой конечно порожденной подгруппе.

УтвеРжДЕНИЕ 2. Для парадоксалъной группь $G$ существует конечно порожденная подгруппа $H$ такая, что $\tau(G)=\tau(H)$.

УтвержДЕниЕ 3 (Джонсон, Деккер, см. [60]). Для парадоксалъной группь $G$ равенство $\tau(G)=4$ справедливо тогда и только тогда, когда $G$ содержит свободную подгруппу $F_{2}$ с двумя образующими.

Это утверждение было получено Джонсоном в 40-х годах прошлого века и является частным случаем результата Деккера, опубликованного в 50-х годах. Подробные ссылки даны в [60].

По утверждению 2 свободная группа $F_{2}$ с двумя образующими относится к классу парадоксальных групп с минимальным числом Тарского, равным 4.

УтвержДЕниЕ 4 (Григорчук, Чеккерини-Зилберштейн, де ля Арп, 1999 г., [24]). Для периодической группь $G$ имеет место ощенка $\tau(G) \geqslant 6$.

Оказалось, что для периодических групп не существует оценки сверху числа Тарского.

УтвеРждЕниЕ 5 (Григорчук, Чеккерини-Зилберштейн, де ля Арп, 1999 г., [24]). Существует неаменабельная периодическая группа со сколь угодно большим числом Тарского.

Вместе с тем для некоторых классов групп такие оценки существуют. На основании результата Адяна [1] о поведении короста группы Бернсайда $B(m, n)$ при $m \geqslant 2$ и нечетных $n \geqslant 665$ была дана оценка для числа Тарского.

УтвеРждениЕ 6 (Григорчук, Чеккерини-Зилберштейн, де ля Арп, 1999 г., [24]). Для группь Бернсайда $B(m, n)$ при $m \geqslant 2$ и нечетных $n \geqslant 665$ число Тарского заключено между 6 и 14.

В данном утверждении указаны оценки, но какие значения числа Тарского реализуются, остается неизвестным. В этом направлении имеется следующий результат.

УтвеРжДЕНИЕ 7 (Ершов, Голан, Сапир, 2014 г., [26]). Существуют неаменабельные группы с числами Тарского 5 и 6.

Интересен вопрос об оценках числа Тарского в зависимости от минимального числа образующих в группе. Оказывается, что таких оценок нет.

УтвержДЕНИЕ 8 (Ершов, Голан, Сапир, 2014 г., [26]). Существуют неаменабельные группь с двумя образующими, обладающие сколь угодно большими числами Тарского. 
И наконец, приведем результат о распределении чисел Тарского.

УтверЖДЕНИЕ 9 (Ершов, Голан, Сапир, 2014 г., [26]). Для любого достаточно большого $n$ существует неаменабельная группа с числом Тарского между $n$ и $2 n$.

Для более детального ознакомления с вопросами, касающимися аменабельности, можно обратиться к работам [22]-[24], [29], [32], [52], [57].

Вопрос, каждому ли натуральному числу, большему четырех, соответствует парадоксальная группа с таким числом Тарского, остается открытым.

Конечные и абелевы группы являются аменабельными группами. В силу замкнутости множества аменабельных групп относительно операций (i)-(iv) разрешимые группы также будут аменабельными.

Определим следующие классы групп:

$\mathscr{K}$ - класс коммутативных групп;

,$\widehat{\aleph}$ - классы нильпотентных и почти нильпотентных групп (групп с нильпотентными подгруппами конечного индекса) соответственно;

$\mathscr{N}_{P}$ - класс групп, не содержащих свободных подполугрупп с двумя образующими;

$\mathscr{S}, \widehat{\mathscr{S}}$ - класс разрешимых и почти разрешимых групп (групп с разрешимыми подгруппами конечного индекса) соответственно;

$\mathscr{E}$ - класс элементарных аменабельных групп (это наименьший класс групп, содержащий все конечные и абелевы группы и замкнутый относительно операций (i)-(iv));

$\mathscr{A}$ - класс аменабельных групп;

$\mathscr{N}$ - класс групп, не содержащих свободных подгрупп с двумя образующими; $\mathscr{W}$ - класс всех групп.

Через $\mathscr{K}_{f}, \aleph_{f}, \widehat{\aleph}_{f}, \mathscr{N}_{P f}, \mathscr{S}_{f}, \widehat{\mathscr{S}}_{f}, \mathscr{E}_{f}, \mathscr{A}_{f}, \mathscr{N}_{f}, \mathscr{W}_{f}$ обозначим подклассы конечно порожденных групп в классах $\mathscr{K}, \aleph, \widehat{\aleph}, \mathscr{N}_{P}, \mathscr{S}, \widehat{\mathscr{S}}, \mathscr{E}, \mathscr{A}, \mathscr{N}, \mathscr{W}$ соответственно.

Справедлива цепочка вложений

$$
\mathscr{K} \subset \widehat{\aleph} \subset \widehat{\mathscr{S}} \subset \mathscr{E} \subseteq \mathscr{A} \subseteq \mathscr{N} \subset \mathscr{W}
$$

ПроБЛема ДэЯ (1957 г., [23]). Являются ли вложения в иепочке

$$
\mathscr{E} \subseteq \mathscr{A} \subseteq \mathscr{N}
$$

строгими?

Была выдвинута гипотеза: дискретная группа $G$ либо аменабельна, либо содержит свободную подгруппу с двумя образующими, т.е.

$$
\mathscr{A}=\mathscr{N} .
$$

Подобное свойство будем называть дихотомией или экстремальным свойством.

Для линейных групп такую гипотезу (и даже большее) доказал в 1979 г. Титс [58] (альтернатива Титса): конечно порожденная линейная группа либо 
содержит свободную подгруппу с двумя образующими, либо является почти разрешимой.

Таким образом, альтернатива Титса, справедливая для линейных групп, поддерживала выдвинутую гипотезу.

Адян [2], Ольшанский [43], Громов [34] (1980-1988 гг.) опровергли гипотезу и построили примеры конечно порожденных групп из $\mathscr{N} \backslash \mathscr{A}$, т. е. построили примеры неаменабельных конечно порожденных групп (не являющихся конечно определенными), не содержащих свободных подгрупп с двумя образующими.

Строгость первого вложения в (2) была доказана Григорчуком в 1984 г. [30]. Он построил пример конечно порожденной группы из $\mathscr{A} \backslash \mathscr{E}$ (әруппа Григорчука). Группа Григорчука также не является конечно определенной (представимой). Позже он же в [31] построил пример конечно определенной группы из $\mathscr{A} \backslash \mathscr{E}$.

Таким образом, для абстрактных групп было показано, что цепочка вложений (2) является строгой. Все приведенные результаты можно сформулировать в терминах вложений и соответствий классов групп, порожденных числом Тарского.

ПреДложениЕ 1. Справедливы следующие утверждения.

1) Имеет место цепочка вложений классов групп

$$
\mathscr{K} \subset \widehat{\aleph} \subset \widehat{\mathscr{S}} \subset \mathscr{E} \subset \mathscr{A} \subset \mathscr{N} \subset \mathscr{W} .
$$

2) Имеются соответствия классов групп, порожденные числом Тарского:

\begin{tabular}{|c|c|c|c|}
\hline число Тарского $\tau(G)$ & $\infty$ & $\ldots, 7,6,5$ & 4 \\
\hline классъ групп & $\mathscr{A}$ & $\mathscr{N} \backslash \mathscr{A}$ & $\mathscr{W} \backslash \mathscr{N}$ \\
\hline
\end{tabular}

Из приведенных соответствий следует, что число Тарского может служить для классификации только лишь среди парадоксальных групп.

Хотелось бы иметь пример конечно порожденной, но при этом конечно определенной группы из $\mathscr{N} \backslash \mathscr{A}$. Так как число Тарского убывает с расширением группы (утверждение 1 ), то это означает поиск группы $G$, максимально близкой к барьеру $\tau(G)=5$. Потенциальным кандидатом на пример такой группы выступает группа Ричарда Томпсона (1965 г.): $F$ - множсество кусочно линейных гомеоморфизмов отрезка $[0,1]$, имеющих изломы лишь в конечном числе двоччно рачиональных точек, а на интервалах дифферениируемости имеющих производную, равную степени двойки [21].

Такая группа $F$ изоморфна группе с двумя образующими и двумя соотношениями

$$
F=\left\langle a, b:\left[a b^{-1}, a^{-1} b a\right],\left[a b^{-1}, a^{-2} b a^{2}\right]\right\rangle
$$

и может быть реализована как группа гомеоморфизмов прямой $\mathbb{R}$. Образующие гомеоморфизмы $\mathbb{R}$ имеют вид, указанный на рис. 1. 


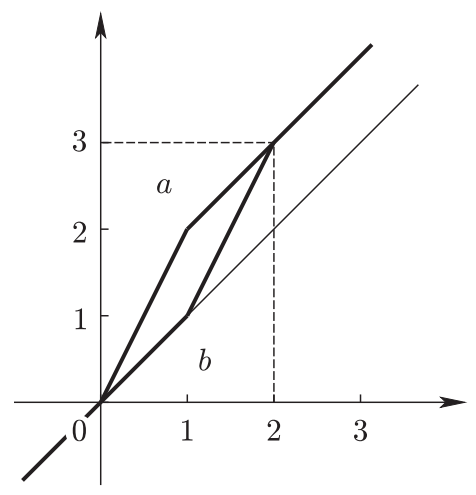

Рис. 1

В $[20]$ показано, что группа $F$ не элементарная и не содержит свободной подгруппы с двумя образующими, т. е. $F \in \mathscr{N} \backslash \mathscr{E}$.

Вопрос. Какая из альтернатив

a) $F \in \mathscr{A} \backslash \mathscr{E}(F-$ аменабельная $)$;

b) $F \in \mathscr{N} \backslash \mathscr{A}(F-$ неаменабельная $)$

справедлива?

По мнению автора, ответ на этот вопрос следует искать на пути систематического исследования групп гомеоморфизмов прямой, их метрических инвариантов и топологических характеристик.

По вопросам аменабельности, парадоксальности, а также других специальных свойств групп читатель может обратиться к работам [2], [21]-[24], [29], [32], [34], [52], [57], [60].

\section{2. Рост конечно порожденной группы и соответствия классов групп}

Важной характеристикой конечно порожденной группы $G=\left\langle g_{1}, \ldots, g_{s}\right\rangle$ является ее рост, определенный по следующему правилу:

$$
\lambda(G)=\lim _{n \rightarrow \infty} \sqrt[n]{\gamma_{G}(n)}
$$

где

$\gamma_{G}(n)=\sharp\left\{g: g=g_{i_{1}}^{\varepsilon_{1}}, \ldots, g_{i_{m}}^{\varepsilon_{m}}, m \leqslant n, i_{j} \in\{1, \ldots, s\}, \varepsilon_{j}=+(-) 1, j=1, \ldots, m\right\}$.

Если $\lambda(G)>1$, то группа имеет экспоненциальный рост; если $\lambda(G)=1$, то группа имеет субэкспоненииальный рост. Если для группы $G=\left\langle g_{1}, \ldots, g_{s}\right\rangle$ функция $\gamma_{G}(n)$ по переменной $n$ растет быстрее, чем любая полиномиальная функция, но медленнее, чем экспоненциальная, то такая группа называется группой промежуточного роста.

Для конечно порожденной группы $G=\left\langle g_{1}, \ldots, g_{s}\right\rangle$ рост $\lambda(G)$ всегда определен, а свойство иметь субэкспоненциальный рост либо промежуточный рост не зависит от выбранной системы образующих. 
Неаменабельные конечно порожденные группы имеют экспоненциальный рост. Следовательно, рост группы может служить для классификации только лишь среди аменабельных конечно порожденных групп. К сожалению, внутри множества $\mathscr{A}$ конечно порожденных аменабельных групп нет соответствий классов групп и роста групп из этих классов. Тем не менее для отдельных классов конечно порожденных групп имеет место взаимно однозначное соответствие.

Теорема 2 (Громов, 1981 г., [35]). Конечно порожденная группа имеет полиномиальный рост тогда и только тогда, когда она почти нильпотентна.

Учитывая значимость свойства почти нильпотентности группы в связи с его однозначным соответствием со свойством полиномиальности роста (теорема 2), представляется важным найти признаки почти нильпотентности группы. Ранее такой результат был получен для разрешимых групп.

Теорема 3 (Розенблатт, 1974 г., [48]). Конечно порожденная разрешимая группа без свободных подполугрупп с двумя образующими почти нильпотентна (соответственно, имеет полиномиальный рост).

В силу теоремы 3 для конечно порожденных групп важно оценить значимость условия существования (отсутствия) свободных подполугрупп с двумя образующими при формировании классов групп. С одной стороны, конечно порожденные группы, содержащие свободные подполугруппы с двумя образующими, имеют экспоненциальный рост (это доказывается с помощью правила пинг-понга, восходящего к Клейну), но среди таких групп могут быть и элементарные группы. В частности, афбинная группа гомеоморфизмов прямой $G=\langle t+1,2 t\rangle$ является разрешимой группой и имеет свободную подполугруппу с двумя образующими (соответственно, имеет экспоненииальный рост).

С другой стороны, существуют примеры конечно порожденных неаменабельных групп (соответственно, экспоненциального роста), не содержащих свободных подполугрупп с двумя образующими. Следовательно, отсутствие свободных подполугрупп с двумя образующими также не может быть признаком ни аменабельности, ни экспоненциального роста группы. Вместе с тем большинство известных групп с экспоненциальным ростом содержат свободные подполугруппы с двумя образующими.

Из теоремы 2 следует, что для группы Григорчука рост более чем полиномиальный. В действительности, для группы Григорчука справедливо утверждение о промежуточном росте.

Теорема 4 (Григорчук, 1984 г., [30]). Для группы Григорчука рост более чем полиномиальный и менее чем экспоненииальный.

Все приведенные результаты можно сформулировать в терминах вложений и соответствий для классов групп, порожденных ростом группы.

ПРЕДЛОЖЕНИЕ 2. Справедливы следующие утверждения.

1) Имеет место цепочка вложений классов конечно порожденных групп

$$
\mathscr{K}_{f} \subset\left[\mathscr{N}_{P f} \cap \mathscr{S}_{f}\right] \subseteq \widehat{\aleph}_{f} \subset \widehat{\mathscr{S}_{f}} \subset \mathscr{E}_{f} \subset \mathscr{A}_{f} \subset \mathscr{N}_{f} \subset \mathscr{W}_{f} .
$$


2) Имеются соответствия классов конечно порожденных групп, порожденные ростом группь:

\begin{tabular}{|c|c|}
\hline рост группь $\lambda(G)$ & классы групп \\
\hline полиномиальный & $\widehat{\aleph}_{f}$ \\
\hline промежуточный и экспоненциалъный & $\mathscr{A}_{f} \backslash \widehat{\aleph}_{f}$ \\
\hline экспоненциалъный & $\mathscr{W}_{f} \backslash \mathscr{A}_{f}$ \\
\hline
\end{tabular}

На этом пути естественно поставить вопрос о выделении классов специальных групп, для которых такие характеристики, как рост группы и наличие (отсутствие) свободных подполугрупп (подгрупп) с двумя образующими, определяли бы их важнейшие свойства. Примером таких специальных классов групп могут служить группы гомеоморфизмов прямой (окружности). Для них ответы на поставленные вопросы можно искать на пути систематического исследования групп гомеоморфизмов прямой (окружности), их метрических инвариантов и топологических характеристик.

\section{3. О реализациях абстрактных групп как групп действий на прямой (окружности)}

Следующим важным аспектом является возможность реализации абстрактных групп и, в частности, их реализация в виде подгрупп группы гомеоморфизмов интервала, прямой, окружности.

Теорема 5 (Григорчук, Гиз, 1996 г., [28]). Счетная группа может быть реализована как группа гомеоморфизмов прямой, сохраняющих ориентацию, тогда и только тогда, когда она правоупорядочиваема.

Более того, такие реализации упорядочиваемых счетных групп в виде групп гомеоморфизмов прямой обладают дополнительным важным свойством: графики различных элементов образуют кортеж, т. е. график одного из них расположен над графиком другого, с возможным касанием.

Теорема 5 указывает на то, что группы гомеоморфизмов прямой (окружности) образуют представительный класс групп в множестве всех абстрактных групп. Исходя из конструкции группы Григорчука был построен первый нетривиальный пример группы из Homeo+ $([0,1])$ как результат вложения группы, ассоциированной с группой Григорчука и также имеющей промежуточный рост.

Заметим, что группа Нотео+ $([0,1])$ гомеоморфизмов интервала $[0,1]$ топологически сопряжена некоторой подгруппе $\mathrm{Homeo}_{+}(\mathbb{R})$ гомеоморфизмов прямой. Для этого определим гомеоморфизм $\eta:(0,1) \rightarrow \mathbb{R}$. Более того, отображение $\eta$ можно определить как диффеоморфизм класса $C^{\infty}$. Очевидно, что группа $\eta \circ \mathrm{Homeo}_{+}([0,1]) \circ \eta^{-1}$ будет подгруппой группы $\mathrm{Homeo}_{+}(\mathbb{R})$, имеющей некоторые дополнительные свойства. 
Теорема 6 (Григорчук, Маки, 1993 г., [33]). Существует конечно порожденная подгруппа группъ Нотео $([0,1])$ гомеоморфизмов интервала $[0,1]$, сохраняющих ориентацию, имеющая промежуточныи рост.

В силу наличия отмеченной топологической сопряженности теорема 6 еще раз указывает на сложность структуры групп гомеоморфизмов прямой.

В действительности можно доказать более сильное, чем теорема 6, утверждение, позволяющее реализовать вложение группы промежуточного роста, ассоциированной с группой Григорчука, в меньшее пространство Diff ${ }_{+}^{1}([0,1])$. А именно, справедлива следующая теорема.

Теорема 7 (Навас, 2004 г., [25]). Существует конечно порожденная подгруппа группы $\operatorname{Diff}_{+}^{1}([0,1])$ с промежуточным ростом.

Интересен вопрос о возможности реализации абстрактных групп в виде подгрупп групп диффеоморфизмов различной гладкости.

ТЕОРема 8 (Фарб, Фрэнкс, 2003 г., [27]). Любая конечно порожденная нильпотентная группа без кручения может быть вложена в $\operatorname{Diff}_{+}^{1}([0,1])$.

Оказывается, что имеет место ограничение на гладкость образа вложения из теоремы 8. Чтобы сформулировать соответствующее утверждение, определим пространства $\operatorname{Diff}_{+}^{1+\alpha}([0,1]), \alpha>0$, следующим образом: $g \in \operatorname{Diff}_{+}^{1+\alpha}([0,1])$ тогда и только тогда, когда существует константа $C_{g}>0$ такая, что при всех $t_{1}$, $t_{2} \in[0,1]$ для производной справедлива оценка $\left|g^{\prime}\left(t_{2}\right)-g^{\prime}\left(t_{1}\right)\right| \leqslant C_{g}\left|t_{2}-t_{1}\right|^{\alpha}$.

Теорема 9 (Деруан, Клепцын, 2004 г., [25]). Любая конечно порожденная без кручения нильпотентная группа ступени $k$ может быть вложена 8 $\operatorname{Diff}_{+}^{1+\alpha}([0,1])$ для любого $\alpha<1 /(k-1)$.

Мы видим, что нильпотентные группы ступени $k>1$ вкладываются в пространства $\operatorname{Diff}_{+}^{1+\alpha}([0,1])$, где $\alpha$ удовлетворяет ограничениям $\alpha<1 /(k-1) \leqslant 1$. Оказывается, что верхнее ограничение $\alpha=1$ является точным.

Теорема 10 (Планте, Тёрстон, 1976 г., [46]). Нилъпотентные подгруппь группъ $\operatorname{Diff}_{+}^{2}([0,1])$ являются коммутативными.

Для групп диффеоморфизмов интервала $[0,1]$ гладкости, большей единицы, существует критерий их почти нильпотентности.

Теорема 11 (Навас, 2007 г., [41]). Для любого заданного $\alpha>0$ каждая конечно порожденная подгруппа группљ $\operatorname{Diff}_{+}^{1+\alpha}([0,1])$ почти нильпотентна $и$, соответственно, является группой полиномиального роста тогда и только тогда, когда она не содержит свободной подполугруппы с двумя образующими.

Стоит отметить очевидное следствие из теоремы 11.

СлеДСТвИЕ 1. Ни для какого $\alpha>0$ не существует конечно порожденных nодгрупп группы $\operatorname{Diff}_{+}^{1+\alpha}([0,1])$, имеющ,и промежуточный рост.

Отсюда следует, что группа, ассоциированная с группой Григорчука, не допускает реализации в виде группы диффеоморфизма интервала $[0,1]$ гладкости выше единицы. Рост группы диффеоморфизмов интервала $[0,1]$ гладкости 
больше единицы обладает экстремальным свойством: он либо полиномиальный, либо экспоненциальный.

Понимание взаимосвязи отмеченных результатов может быть уточнено благодаря систематическому исследованию групп гомеоморфизмов прямой (окружности), их метрических инвариантов и топологических характеристик.

Большая тема, связанная с вопросами аппроксимации действия групп, в частности вопросы финитной аппроксимации и свободной периодической аппроксимации, в данном обзоре не рассматривается. По всем этим вопросам можно обратиться к работам [55], [56], [59].

\section{4. Топологические характеристики и метрические инварианты групп гомеоморфизмов прямой (окружности)}

В дальнейшем через $\mathrm{Homeo+}_{+}(\mathbb{X}), \mathbb{X}=\mathbb{R}, \mathbb{S}^{1}$, будем обозначать группу всех гомеоморфизмов $\mathbb{X}$, сохраняющих ориентацию. Если в ссылке указаны две работы, то первой идет работа, где впервые был получен результат, а вторым идет обзор, где собраны все результаты данной направленности. Определим важное подмножество $G^{S}$ группы $G \subseteq$ Homeo+ $_{+}(\mathbb{X})$ как объединение стабилизаторов:

$$
G^{S}=\bigcup_{t \in \mathbb{X}} \operatorname{St}_{G}(t) .
$$

Множество $G^{s}$ может и не быть группой. Очевидны вложения

$$
G^{S} \subseteq\left\langle G^{S}\right\rangle \subseteq G
$$

Оказывается, такая цепочка вложений характеризуется экстремальным свойством, описанным в нижеследующей лемме.

Лемма 1 [6], [14]. Пусть $G \subseteq$ Homeo+ $_{+}(\mathbb{R})$. Тогда или $G^{S}=\left\langle G^{S}\right\rangle$, или $\left\langle G^{S}\right\rangle=G$.

Альтернатива, сформулированная в лемме, является нестрогой, так как существуют группы, для которых $G^{S}=G$. Приведенная лемма лежит в основе важной теоремы о факторгруппе.

Теорема 12 (Бекларян, 1993 г., [6], [14]). Пусть $G \subseteq$ Homeo $_{+}(\mathbb{X})$. Тогда факторгруппа $G /\left\langle G^{S}\right\rangle$ коммутативна и изоморфна некоторой подгруппе аддитивной группы $\mathbb{X}$.

ЗАмечАниЕ 1 . Пусть $G \subseteq$ Homeо+ $_{+}(\mathbb{X})$. Если $G /\left\langle G^{S}\right\rangle \neq\langle e\rangle$, то в группе $G$ существует свободно действующий элемент.

Предыдущая теорема 12 о факторгруппе является определяющей при исследовании групп гомеоморфизмов прямой (окружности). В частности, показано, что из нетривиальности факторгруппы $G /\left\langle G^{s}\right\rangle$ следует существование $G$-инвариантной меры. Вместе с тем такая характеристика, как факторгруппа $G /\left\langle G^{S}\right\rangle$, не может быть универсальным инструментом при классификации групп гомеоморфизмов прямой, так как она является нетривиальной только 
лишь для групп с инвариантной мерой (см. теорему 22 ниже). В частности, аффинная группа $G=\langle t+1,2 t\rangle$ с двумя образующими является разрешимой (элементарной), но факторгруппа $G /\left\langle G^{S}\right\rangle$ тривиальна и не несет в себе никакой информации об исходной группе $G$.

В основе утверждения теоремы 12 лежит некоторое естественное отношение частичного порядка для групп гомеоморфизмов прямой, сохраняющих ориентацию [8], и фундаментальная теорема Гёльдера об архимедовых группах [39].

Несколько замечаний по истории вопроса. Для некоторых специальных групп гомеоморфизмов прямой, сохраняющих ориентацию, утверждение теоремы о факторгруппе было получено в работах [42], [49]-[51], [53]. Новиков в [42] рассматривал свободно действующие группы. Солодов в [53] рассматривал конечно порожденные группы, не содержащие свободных подполугрупп с двумя образующими. Сали в [49]-[51] рассматривал счетные группы $G$ с общей неподвижной точкой для элементов, имеющих хотя бы одну неподвижную точку. Более детальное обсуждение этого вопроса можно найти в обзоре [14].

4.1. Минимальные множества. Для группы $G \subseteq \mathrm{Homeo}_{+}(\mathbb{X})$ важной топологической характеристикой является минимальное множество.

ОПРЕДЕЛЕНИЕ 4. Минимальное множество группы $G \subseteq \operatorname{Homeo}(\mathbb{X})$ - это замкнутое $G$-инвариантное подмножество $\mathbb{X}$, не содержащее собственных замкнутых $G$-инвариантных подмножеств. Если не существует непустого минимального множества, то по определению будем полагать, что оно пустое.

Важность минимальных множеств состоит в том, что они определяют носители метрических инвариантов.

Другой важнейшей топологической характеристикой является множество

$$
\operatorname{Fix} G^{S}=\left\{t \in \mathbb{X}: \forall g \in G^{S} g(t)=t\right\} .
$$

Теорема 13 (Бекларян, 1996 г., [10], [14]). Пусть $G \subseteq$Homeo+ $\left._{+} \mathbb{X}\right)$. Тогда справедливо одно из следующих взаимоисключающих утверждений:

а) любое минимальное множество дискретно и принадлежит множеству $\operatorname{Fix} G^{S}$, а множество $\operatorname{Fix} G^{S}$ состоит из оббединения минимальных множеств $E_{\alpha}, \alpha \in \mathscr{A}$, m.e. Fix $G^{S}=\bigcup_{\alpha \in \mathscr{A}} E_{\alpha}$;

b) минимальное множество единственно, является совершенным нигде не плотным подмножеством $\mathbb{R}$, содержится в замыкании орбиты $\overline{G(t)}$ произвольной точки $t \in \mathbb{X}($ и обозначается через $E(G))$;

c) минимальное множество совпадает с пространством $\mathbb{X}$ (и также обозначается через $E(G))$;

d) минимальное множество пустое (в случае $\mathbb{X}=\mathbb{R})$.

При $\mathbb{X}=\mathbb{S}$ минимальное множество всегда непусто. В случае $\mathbb{X}=\mathbb{R}$ для формулировки признака существования непустого минимального множества определим подмножество

$$
G_{\infty}^{S}=\left\{g \in G^{S}: \sup \{t: g(t)=t\}=+\infty, \inf \{t: g(t)=t\}=-\infty\right\} .
$$




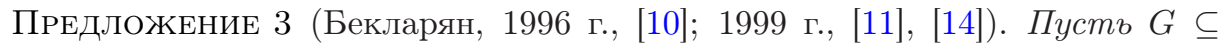
Hотео $_{+}(\mathbb{R})$ и выполняется хотя бы одно из условий:

a) $G$ - конечно порожденная группа;

b) $\operatorname{Fix} G^{S} \neq \varnothing$;

c) $G \neq G_{\infty}^{S}$.

Тогда существует непустое минимальное множество.

Приведенный признак существования непустого минимального множества основан на структурных свойствах группы гомеоморфизмов прямой. Приведем критерий существования непустого минимального множества, основанный на локальных свойствах гомеоморфизмов.

ПредложениЕ 4 (Планте, 1983 г., [45]; Сали, 1982-1985 гг., [49]-[51]). Пусть $G \subseteq$ Homeo+ $\left._{(} \mathbb{R}\right)$ - счетная группа. Если $G$ абелева и ее элементы принадлежат $\operatorname{Diff}^{2}(\mathbb{R})$ или элементы $G$ задаются аналитическими функииями, то существует непустое минимальное множество.

Существуют примеры счетных абелевых групп гомеоморфизмов прямой, сохраняющих ориентацию, для которых минимальное множество пусто.

Важно знать наследственные свойства минимальных множеств.

Теорема 14 (Бекларян, 1999 г., [11], [14]). Пусть $G \subseteq \mathrm{Homeo}_{+}(\mathbb{X})$. Если для подгруппы $\Gamma \subseteq G$ минимальное множество $E(\Gamma)$ не пусто и не дискретно, mo $E(\Gamma) \subseteq E(G)$.

Дадим усиление этой теоремы для нормальных подгрупп.

Теорема 15 (Бекларян, 1999 г., [11], [14]). Пусть $G \subseteq \mathrm{Homeo}_{+}(\mathbb{X})$. Если для нормальной подгруппы $\Gamma \subseteq G$ минимальное множество $E(\Gamma)$ не пусто и не дискретно, то оно совпадает с минимальным множеством исходной группъ $G$, m.е. $E(\Gamma)=E(G)$.

Как видим, минимальные множества нормальных подгрупп исходной группы $G \subseteq \mathrm{Homeo}_{+}(\mathbb{X})$ обладают экстремальным свойством: они или дискретны (возможно, пусты), или совпадают с минимальным множеством исходной группы $G$.

В условиях теоремы 15 нормальная подгруппа $\Gamma$ и исходная группа $G$ имеют одно и то же минимальное множество. Этот факт особенно полезен в случае, когда для исходной группы существует алгебраически более простая подгруппа, имеющая то же минимальное множество, что и исходная группа.

Определим одну важную подгруппу исходной группы, связанную с минимальным множеством.

ОПредЕлЕНиЕ 5. Для группы $G \subseteq \operatorname{Homeo}_{+}(\mathbb{X})$ нормальная подгруппа $H_{G}$ определяется следующим образом:

1) если минимальное множество не пусто и не дискретно, то положим

$$
H_{G}=\{h \in G: E(G) \subseteq \operatorname{Fix}\langle h\rangle\} ;
$$


2) если минимальное множество не пусто и дискретно, то положим $H_{G}=G^{S}$ (из дискретности минимального множества следует непустота множества $\operatorname{Fix} G^{S}$, из непустоты $\operatorname{Fix} G^{S}$ следует, что $G^{S}$ является нормальной подгруппой);

3) если минимальное множество пусто, то положим $H_{G}=\langle e\rangle$.

Заметим, что если минимальное множество совпадает со всей прямой, то $H_{G}=\langle e\rangle$.

Несколько замечаний по истории вопроса. K ранним исследованиям по минимальным множествам относятся работы Данжуа для циклической группы диффеоморфизмов окружности гладкости $C^{(2)}$. В частности, им был получен первый результат об отсутствии минимального множества, изоморфного канторову множеству, который известен под названием теоремы Данжуа [38]. Изучение структуры минимального множества для произвольной группы гомеоморфизмов окружности $G \subseteq \mathrm{Homeo}_{+}\left(\mathbb{S}^{1}\right)$ началось значительно позже. Наиболее ранней работой, затрагивающей этот вопрос, является курс лекций Альфорса [4]. Этот вопрос весьма важен и для произвольной группы гомеоморфизмов прямой $G \subseteq$ Homeo+ $_{+}(\mathbb{R})$ [12], [13]. В отличие от групп гомеоморфизмов окружности, в случае прямой возникает дополнительная трудность, связанная с непустотой минимального множества. Непустота минимального множества устанавливается и для некоторых групп диффеоморфизмов прямой в работах [45], [49]--[51]. Справедливость же теоремы Данжуа следует ожидать только при дополнительных ограничениях на группу гомеоморфизмов прямой (окружности). В частности, такая теорема получена для одного важного класса квазисимметрических групп [12], а также для групп диффеоморфизмов интервала $C^{(1+\alpha)}([0,1]), \alpha>0$ [41]. Более детальное обсуждение этого вопроса можно найти в обзоре [14].

4.2. Метрические инварианты. Свойства, связанные с $\omega$-проективно инвариантными мерами, могут быть положены в основу подхода к классификации групп гомеоморфизмов прямой (окружности). Это объясняется тем, что такие меры оказываются тесно связанными с характеристиками групп гомеоморфизмов прямой (окружности) различной природы: топологическими, алгебраическими, а также комбинаторными. Далее будут определены как $\omega$-проективно инвариантные меры групп гомеоморфизмов прямой (окружности), так и их связи с указанными характеристиками.

Определим важнейший метрический инвариант в виде $\omega$-проективно инвариантной меры. Пусть $\mathscr{M}$ обозначает пространство зарядов на $\mathbb{X}$, конечных на компактах $\left(\mathscr{M}^{+}-\right.$конус борелевских мер). В случае $\mathbb{X}=\mathbb{R}$ пространство $\mathscr{M}$ рассматривается как сопряженное пространство к пространству $\mathscr{R}(\mathbb{R})$ непрерывных функций на $\mathbb{R}$ с компактным носителем и топологией индуктивного предела $\mathscr{R}(\mathbb{R})$ [47]. В случае $\mathbb{X}=\mathbb{S}^{1}$ пространство $\mathscr{M}$ рассматривается как сопряженное пространство к пространству $C\left(\mathbb{S}^{1}\right)$ непрерывных функций на $\mathbb{S}^{1}$. Для группы $G \subseteq \mathrm{Homeo}_{+}(\mathbb{X})$ через $G_{*}$ будем обозначать изоморфную ей группу аффинных действий на конусе $\mathscr{M}^{+}$. Изоморфизм $\theta: G \rightarrow G_{*}$, где $\theta(g)=g_{*}$, определяется следующим образом: для любой меры $\mu$ и любого борелевского 
множества $B$

$$
g_{*} \mu(B)=\mu\left(g^{-1}(B)\right) .
$$

Заметим, что конус положительных мер $\mathscr{M}^{+}$инвариантен относительно группы аффинных действий $G_{*}$. Для любой меры $\mu \in \mathscr{M}^{+}$через $\mathscr{K}_{\mu}(G)$ обозначим замкнутый выпуклый конус, порожденный орбитой $G_{*}(\mu)=\left\{g_{*} \mu: g \in G\right\}$ меры $\mu$, т. е.

$$
\mathscr{K}_{\mu}(G)=\overline{\operatorname{conv}\left\{\lambda g_{*} \mu: g \in G, \lambda \in \mathbb{R}_{+}\right\}} .
$$

Очевидно, что конус $\mathscr{K}_{\mu}(G)$ инвариантен относительно группы непрерывных аффинных действий $G_{*}$.

ОПРеДЕЛЕНиЕ 6. Пусть $G \subseteq \mathrm{Homeo}_{+}(\mathbb{X})$, а $\mu \in \mathscr{M}^{+}$. Конус $\mathscr{K}_{\mu}(G)$ называется минимальным, если для любой меры $\bar{\mu} \in \mathscr{K}_{\mu}(G)$ выполнено условие $\mathscr{K}_{\mu}(G)=\mathscr{K}_{\bar{\mu}}(G)$.

Пусть $\mathscr{K} \subseteq \mathscr{M}^{+}{ }_{-}$конус и $\mu \in \mathscr{K}$. Луч $\lambda \mu, \lambda>0$, называется крайним, если не существует мер $\mu_{1}, \mu_{2} \in \mathscr{K} \backslash \mu$ и неотрицательных чисел $\lambda_{1}, \lambda_{2}$ таких, что $\mu=\lambda_{1} \mu_{1}+\lambda_{2} \mu_{2}$.

ОПредЕЛЕниЕ 7. Борелевская мера $\mu \in \mathscr{M}^{+}$, конечная на компактах, называется $\omega$-проективно инвариантной относительно группы $G \subseteq \operatorname{Homeo}_{+}(X)$, если выпуклый конус $\mathscr{K}_{\mu}(G)$ является минимальным, луч $\lambda \mu$ крайним, а $\omega$ является мощностью множества крайних лучей.

В случае $\omega=1$ инвариантный конус $\mathscr{K}_{\mu}(G)$ одномерный и такая мера называется проективно инвариантной мерой. Если инвариантный конус $\mathscr{K}_{\mu}(G)$ не только одномерный, но и неподвижный относительно группы аффинных действий $G_{*}$, то проективно инвариантная мера называется инвариантной. Очевидно, что в случае окружности $\mathbb{X}=\mathbb{S}^{1}$ всякая проективно инвариантная мера является инвариантной. Свойства инвариантной или проективно инвариантной меры тесно связаны с минимальными множествами. В частности, будет показано, что для группы с инвариантной или проективно инвариантной мерой минимальное множество непусто. Сформулируем признак существования $\omega$-проективно инвариантной меры.

Теорема 16 (Бекларян, 1999 г., [11], [14]). Пустъ $G \subseteq \mathrm{Homeo}_{+}(\mathbb{R})$ и минимальное множество группь $G$ непусто. Тогда найдется такое кардинальное число $\omega$, что для группь $G$ существует $\omega$-проективно инвариантная мера.

Здесь интересен следующий вопрос: в каких случаях существует $\omega$-проективно инвариантная мера с конечным $\omega$ ? Ответ на этот вопрос дает следующая теорема об экстремальном свойстве $\omega$-проективно инвариантной меры.

Теорема 17 (Бекларян, 2004 г., [14]). Пусть $G \subseteq$ Homeo+ $_{+}(\mathbb{R})$, а минимальное множество группь $G$ не пусто и не дискретно. Тогда для $\omega$-проективно инвариантной меры $\mu$ кардинальное число $\omega$ либо равно 1 , либо бесконечно.

Интересен вопрос, при каких условиях из существования $\omega$-проективно инвариантной меры следует существование инвариантной или проективно инвариантной меры? Мы опишем препятствия к существованию инвариантной (или 
проективно инвариантной) меры в терминах топологических характеристик, в смешанных терминах топологических и алгебраических характеристик, либо в терминах комбинаторных характеристик групп гомеоморфизмов.

а) Инвариантные меры. Первым, наиболее значимым, результатом по вопросу существования инвариантной меры является теорема БоголюбоваКрылова-Дэя.

Теорема 18 (Боголюбов-Крылов-Дэй [37], [23]). Для аменабельной груnпъ $G$, действующей гомеоморфизмами на компакте $\mathbb{K}$, существует вероятностная борелевская инвариантная мера.

По истории этого вопроса и важнейшим результатам существует исчерпывающий обзор Д. В. Аносова [5]. Даже для группы гомеоморфизмов окружности теорема Боголюбова-Крылова-Дэя и ее модификации оставались всего лишь признаками существования инвариантной меры, но не критериями. Для групп гомеоморфизмов прямой к наиболее существенным продвижениям в проблематике существования инвариантной меры следует отнести работу [44], в которой для конечно порожденных групп гомеоморфизмов прямой удалось сформулировать критерий существования инвариантной меры в терминах асимптотических характеристик исходной группы.

ОПРЕДЕЛЕНиЕ 8. Для конечно порожденной группы $G \subseteq \mathrm{Homeo}_{+}(\mathbb{R})$ орбита $G(t)$ точки $t \in \mathbb{R}$ имеет неэкспоненциальный рост, если

$$
\lim _{n \rightarrow \infty} \inf \sqrt[n]{\left|G^{n}(t)\right|}=1
$$

где $G^{n}$ - слова длины не более $n$, а $\left|G^{n}(t)\right|$ - мощность множества точек $\{g(t)$ : $\left.g \in G^{n}\right\}$.

Теорема 19 (Планте [44]). Пусть $G \subseteq \mathrm{Homeo+}_{+}(\mathbb{R})$ - конечно порожденная группа. Для существования борелевской меры, конечной на компактах и инвариантной относительно группъ $G$, необходимо и достаточно, чтобъ существовала точка $t \in \mathbb{R}$, орбита которой имеет неэкспоненииальный рост.

K сожалению, такой критерий не может быть распространен на группы, не являющиеся конечно порожденными. Очевидно, что существование инвариантной меры эквивалентно условию непустоты носителя такой меры. На этом пути автору удалось для произвольной группы гомеоморфизмов прямой получить критерий существования инвариантной меры в терминах топологической характеристики $\operatorname{Fix} G^{S}$, формулировка которого приводится в теореме 20.

Теорема 20 (Бекларян, 1993 г., [7], [14]). Пусть $G \subseteq \mathrm{Homeo}_{+}(\mathbb{X})$. Тогда следующие утверждения эквивалентны:

1) существует борелевская (вероятностная в случае $\mathbb{X}=\mathbb{S}^{1}$ ) мера $\mu$, конечная на компактах и инвариантная относительно группы $G$;

2) множество Fix $G^{S}$ непусто.

Непустота замкнутого инвариантного множества Fix $G^{S}$ и является топологическим критерием непустоты носителя меры. Критерий в теореме Планте, 
хотя и формулируется в терминах асимптотических свойств орбиты, в действительности характеризует условие непустоты носителя инвариантной меры.

Для групп гомеоморфизмов окружности критерий существования инвариантной меры может быть дополнен ее формулировкой в терминах гомоморфизмов. Хорошо известно, что для любого гомеоморфизма $g \in \mathrm{Homeo}_{+}\left(\mathbb{S}^{1}\right)$ окружности определен топологический инвариант, называемый числом вращения $\rho(g)$ гомеоморфизма. В таком случае определено отображение $\rho: \mathrm{Homeo}_{+}\left(\mathbb{S}^{1}\right) \rightarrow \mathbb{S}^{1}$.

Теорема 21 (Бекларян, 1996 г., [10], [14]). Пусть $G \subseteq \mathrm{Homeo}_{+}\left(\mathbb{S}^{1}\right)$. Тогда следующие утверждения эквивалентнъ:

1) существует вероятностная борелевская мера $\mu$, инвариантная относительно группь $G$;

2) множество Fix $G^{S}$ непусто;

3) отображение $\rho$ является гомоморфизмом.

Приведем признак существования инвариантной меры в терминах факторгруппы $G /\left\langle G^{S}\right\rangle$, который следует из теоремы о факторгруппе (теорема 12) и теоремы 20 .

Теорема 22 (Бекларян, 1996 г., [9], [14]). Пусть $G \subseteq \mathrm{Homeo}_{+}(\mathbb{X})$. Если факторгруппа $G /\left\langle G^{S}\right\rangle$ нетривиальна, то существует мера $\mu$, конечная на компактах и инвариантная относительно группы $G$.

ЗАмечание 2. Для группы $G \subseteq \mathrm{Homeo}_{+}(\mathbb{R})$, в силу предложения 3 и теоремы 20 , из существования инвариантной меры следует, что минимальное множество непусто.

ЗАмечаниЕ 3 . В силу теоремы 13 , для группы $G \subseteq \mathrm{Homeo+}_{+}(\mathbb{X})$ из существования непустого дискретного минимального множества следует существование борелевской меры $\mu$, конечной на компактах и инвариантной относительно группы $G$.

ЗАмечание 4 . Пусть $G \subseteq \mathrm{Homeo}_{+}(\mathbb{X})$ и для группы $G$ минимальное множество непусто. Тогда для группы $G$ выполняется включение $H_{G} \subseteq G^{S}$. Более того, для существования борелевской меры $\mu$, конечной на компактах и инвариантной относительно группы $G$, необходимо и достаточно, чтобы выполнялось равенство $H_{G}=G^{S}$.

Мы уже отмечали, что такая характеристика, как факторгруппа $G /\left\langle G^{S}\right\rangle$, не может быть универсальным инструментом при классификации групп гомеоморфизмов прямой, так как она оказывается нетривиальной только лишь для групп с инвариантной мерой и свободно действующим элементом. С другой стороны, характеристики в виде нетривиальной факторгруппы $G /\left\langle G^{S}\right\rangle$, как и топологическое условие $\operatorname{Fix} G^{S} \neq \varnothing$ из теоремы 20, не дают никакой информации о группах, не удовлетворяющих указанным признаку или критерию существования инвариантной меры соответственно. Этот недостаток может быть преодолен за счет переформулировки отмеченных критериев в виде альтернатив. 
В терминах комбинаторных характеристик сформулируем критерий существования инвариантной борелевской меры на окружности в виде строгой альтернативы, являющейся неулучшаемым усилением теоремы Боголюбова-Крылова-Дэя о существовании инвариантной меры для аменабельной группы, действующей на окружности.

Теорема 23 (Бекларян, 2002 г., [13], [14]). Для группь гомеоморфизмов окружности $G \subseteq$ Homeо( $\left.\mathbb{S}^{1}\right)$ либо факторгруппа $G / H_{G}$ содержит свободную подгруппу с двумя образующими, либо существует вероятностная борелевская мера, инвариантная относительно группь $G$.

Мы можем вместо сильной альтернативы из теоремы 23 дать формулировку слабой альтернативы, но при этом факторгруппу придется заменить самой исходной группой. Такая слабая альтернатива для группы гомеоморфизмов окружности представляет собой как признак существования инвариантной меры, так и признак существования свободной подгруппы с двумя образующими.

СлеДСтвие 2. Пусть $G \subseteq \operatorname{Homeo}\left(\mathbb{S}^{1}\right)$, тогда или группа $G$ содержит свободную подгруппу с двумя образующими, или существует вероятностная борелевская мера, инвариантная относительно группы $G$.

ДокАзАТЕльство. Действительно, если группа $G$ не содержит свободную подгруппу с двумя образующими, то и факторгруппа $G / H_{G}$ не будет содержать свободную подгруппу с двумя образующими. Тогда по теореме 23 существует вероятностная борелевская мера, инвариантная относительно группы $G$. Следствие доказано.

В работе Солодова [54] была сформулирована следующая альтернатива: nусть $G \subseteq$ Homeо $\left(\mathbb{S}^{1}\right)$, тогда или группа $G$ содержит свободную подгруппу c двумя образующими, или $\operatorname{Fix} G^{S} \neq \varnothing$ и отображение $\rho$, задаваемое числом вращения, является гомоморфизмом.

Вопрос об эквивалентности условий "Fix $G^{S} \neq \varnothing$ ” и "отображение $\rho$ является гомоморфизмом" не изучался. Так как по теореме 21 существование инвариантной меры эквивалентно условию $\mathrm{Fix} G^{S} \neq \varnothing$, то альтернатива, сформулированная Солодовым, эквивалентна слабой альтернативе из следствия 2. Слабая альтернатива, в формулировке следствия 2, была независимо доказана Маргулисом [40] в 2000 г.

Приведем эквивалентную переформулировку теоремы 23 , в которой все утверждения приводятся исключительно в терминах комбинаторных характеристик.

Теорема 24 (Бекларян, 2002 г., [13], [14]). Для группь гомеоморфизмов окружности $G \subseteq$ Homeo( $\left.\mathbb{S}^{1}\right)$ факторгруппа $G / H_{G}$ либо содержит свободную подгруппу с двумя образующими, либо является коммутативной.

В случае групп гомеоморфизмов окружности свойство факторгруппы $G / H_{G}$ может быть использовано для их классификации. Возникает два непересекающихся класса. В одном классе находятся группы с коммутативными фактор- 
группами $G / H_{G}$, в другом - группы с факторгруппами $G / H_{G}$, содержащими свободные подгруппы с двумя образующими. $\mathrm{K}$ сожалению, данный подход не применим к изучению само́й нормальной подгруппы $H_{G}$. Более детальное исследование нормальной подгруппы $H_{G}$ приводит к изучению индуцированных групп гомеоморфизмов прямой, а для них используемое свойство факторгруппы отсутствует. Поэтому задача выявления аналогичных свойств для групп гомеоморфизмов прямой является актуальной.

На этом пути для групп гомеоморфизмов прямой $G \subseteq \mathrm{Homeo}_{+}(\mathbb{R})$ также удается в терминах характеристик факторгруппы $G / H_{G}$ сформулировать критерий существования инвариантной меры. В отличие от критерия существования инвариантной меры для группы гомеоморфизмов окружности, условия на факторгруппу $G / H_{G}$ должны быть более жесткими.

Теорема 25 (Бекларян, 2002 г., [13], [14]). Для группь гомеоморфизмов прямой $G \subseteq \mathrm{Homeo}_{+}(\mathbb{R})$ с непустым минимальным множеством либо факторгруппа $G / H_{G}$ содержит свободную подполугруппу с двумя образующими, либо существует борелевская мера, конечная на компактах и инвариантная относительно группи $G$.

Мы можем вместо сильной альтернативы из теоремы 25 дать формулировку слабой альтернативы, но при этом факторгруппу заменить самой исходной группой. Такая слабая альтернатива для группы гомеоморфизмов прямой представляет собой как признак существования инвариантной меры, так и признак существования свободной подполугруппы с двумя образующими.

СлеДСтвиЕ 3. Для группы гомеоморфизмов прямой $G \subseteq \mathrm{Homeo}_{+}(\mathbb{R})$ с непустым минимальным множеством или группа $G$ содержит свободную подполугруппу с двумя образующими, или существует борелевская мера, конечная на компактах и инвариантная относительно группы $G$.

ДокАЗАТЕЛЬСтво аналогично доказательству следствия 2 с той лишь разницей, что вместо теоремы 23 надо использовать теорему 25.

Приведем эквивалентную переформулировку теоремы 25, в которой все утверждения приводятся исключительно в терминах свойств факторгруппы $G / H_{G}$.

Теорема 26 (Бекларян, 2002 г., [13], [14]). Для группы гомеоморфизмов прямой $G \subseteq$ Homeо $_{+}(\mathbb{R})$ с непустым минимальным множеством факторгруппа $G / H_{G}$ либо содержит свободную подполугруппу с двумя образующими, либо является коммутативной.

Критерии существования метрических инвариантов могут быть переформулированы в терминах полусопряженности, определение которой приводится ниже.

ОПредЕление 9. Пусть заданы группы $G,{ }_{*} G \subseteq$ Homeo $_{+}(\mathbb{R})$. Группа $G$ называется полусопряженной группе ${ }_{*} G$, если существуют сохраняющее ориентацию (монотонно возрастающее) отображение $\eta: \mathbb{R} \rightarrow \mathbb{R}$ с образом, состоящим 
из более чем одной точки, и эпиморфизм $\eta^{\sharp}: G \rightarrow{ }_{*} G$ такие, что для любого $q \in G$ диаграмма

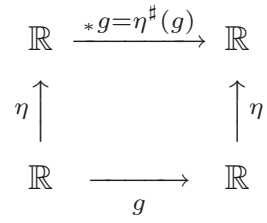

коммутативна, т. е. $\eta^{\sharp}(g) \eta=\eta g$.

Если в определении полусопряженности отображение $\eta$ непрерывно, то говорят о топологической полусопряженности. Если отображение $\eta$ является гомеоморфизмом (и, как следствие, эпиморфизм $\eta^{\sharp}$ является изоморфизмом), то группа $G$ называется топологически сопряженной группе ${ }_{*} G$ или просто conpяженной.

ОПредЕлЕНиЕ 10. Пусть заданы группы $G,{ }_{*} G \subseteq \mathrm{Homeo}_{+}\left(\mathbb{S}^{1}\right)$. Группа $G$ называется полусопряженной группе ${ }_{*} G$, если:

1) группа $\widehat{G} \subseteq \operatorname{Homeo}(\mathbb{R})$ полусопряжена группе $* \widehat{G} \subseteq \operatorname{Homeo}(\mathbb{R})$, где $\widehat{G}$ и ${ }_{*} \widehat{G}-$ группы накрытий для гомеоморфизмов окружности из $G$ и ${ }_{*} G$ соответственно;

2 ) отображение $\eta$ из определения 9 коммутирует с гомеоморфизмом $\bar{g}(t)=$ $t+1$, т. е. $\eta \bar{g}=\bar{g} \eta$.

Сформулируем критерий существования инвариантной меры в терминах полусопряженности.

Теорема 27 (Бекларян, 1996 г., [8], [14]). Пусть $G \subseteq \mathrm{Homeo}_{+}(\mathbb{R})$. Тогда следующие утверждения эквивалентнь:

1) существует борелевская мера $\mu$, конечная на компактах и инвариантная относительно группы $G$;

2) группа $G$ полусопряжена некоторой группе ${ }_{*} G \subseteq \mathrm{Homeo}_{+}(\mathbb{R})$ сдвигов на прямой.

Приведем аналог теоремы 27 для групп, действующих на окружности.

Теорема 28 (Бекларян, 1996 г., [8], [14]). Пусть $G \subseteq \mathrm{Homeo}_{+}\left(S^{1}\right)$. Тогда следующие утверждения эквивалентнъ:

1) существует вероятностная мера $\mu$, инвариантная относительно групnъь $G$;

2) группа $G$ полусопряжена некоторой группе ${ }_{*} G \subseteq \mathrm{Homeo}_{+}\left(\mathbb{S}^{1}\right)$ вращений окружности.

Опишем связь между отображением $\eta$ и инвариантной мерой $\mu$ из теоремы 27.

ЗАмЕчАниЕ 5. В теореме 27 мера $\mu$ и соответствующее отображение $\eta$ из определения полусопряженности связаны соотношением

$$
\eta(t)=\widetilde{\mu}([\bar{t}, t)), \quad \widetilde{\mu}([\bar{t}, t))= \begin{cases}+\mu([\bar{t}, t)), & \text { если } t \geqslant \bar{t}, \\ -\mu((t, \bar{t}]), & \text { если } t<\bar{t},\end{cases}
$$


где $\bar{t} \in \mathbb{R}$ - некоторая заданная точка. Из непрерывности отображения $\eta$ следует непрерывность меры $\mu$, и наоборот.

В терминах полусопряженности подгруппа $H_{G}\left(\right.$ см. замечание $4: H_{G}=G^{S}$ ) совпадает с ядром гомоморфизма $\eta^{\sharp}$, т. е. ker $\eta^{\sharp}=H_{G}$, а факторгруппа $G / H_{G}$ изоморфна группе сдвигов (вращений) ${ }_{*} G$, т. е. ${ }_{*} G \simeq G / H_{G}$.

Для групп гомеоморфизмов прямой свойства факторгруппы $G / H_{G}$ из теоремы 26 могут быть положены в основу классификационной схемы. В силу эквивалентности теорем 25 и 26 множество всех групп с непустым минимальным множеством не только разделяется на два класса (класс групп с инвариантной мерой и класс групп без инвариантной меры), но и содержит дополнительную информацию о факторгруппе $G / H_{G}$. Вместе с тем такая схема обладает рядом недостатков. Факторгруппа $G / H_{G}$, содержащая свободную подполугруппу с двумя образующими, может быть как элементарной (например, разрешимой аффинной группой $G=\langle t+1,2 t\rangle$ ), так и группой, содержащей свободную подгруппу с двумя образующими. Несмотря на эти недостатки, условие непустоты минимального множества и характеристики факторгруппы $G / H_{G}$ (теоремы 25 и 26) разбивают множество всех групп гомеоморфизмов прямой на три класса: группы с инвариантной мерой; группы с непустым минимальным множеством и без инвариантной меры; группы с пустым минимальным множеством. В связи с полученным разбиением возникает задача более детального описания класса групп с непустым минимальным множеством и без инвариантной меры и класса групп с пустым минимальным множеством. В частности, для класса групп с непустым минимальным множеством и без инвариантной меры такое более детальное описание связано с более общим метрическим инвариантом в виде проективно инвариантной меры.

Несколько замечаний по истории вопроса. Вопрос существования инвариантной меры для группы гомеоморфизмов локально компактного пространства является центральным в теории Боголюбова-Крылова. Как уже отмечалось, по истории этого вопроса и важнейшим результатам существует исчерпывающий обзор Д. В. Аносова [5] с полным списком литературы. Несмотря на наличие критерия существования инвариантной меры для произвольной группы гомеоморфизмов прямой, сформулированного в теореме 20, весьма важны переформулировки критерия существования инвариантной меры в терминах и иных свойств группы. В частности, оставался открытым вопрос о существовании инвариантной меры для групп, имеющих инвариантную меру для каждой конечно порожденной подгруппы (удовлетворяющих условию Планте для каждой конечно порожденной подгруппы). Такой критерий также получен автором и приводится в работе [18]. На этом пути критерий существования инвариантной меры был сформулирован и в терминах коммутанта [19]. Ряд других вопросов, связанных с переформулировками критерия существования инвариантной меры в терминах иных свойств группы гомеоморфизмов прямой (окружности), а также описание носителя инвариантной меры и эргодические свойства такой инвариантной меры приводятся в обзоре автора [14]. 
b) Проективно инвариантные меры. Сформулируем важный критерий существования проективно инвариантной меры, использующий как топологические, так и алгебраические характеристики группы преобразований. Для любого элемента $q \in \mathrm{Homeo}_{+}(\mathbb{R})$ будем пользоваться следующими обозначениями:

$$
\begin{aligned}
T_{q} & =\sup \{t: q(t)=t\}, \\
t_{q} & =\inf \{t: q(t)=t\}, \quad \text { если } \operatorname{Fix}\langle q\rangle \neq \varnothing ; \\
T_{q} & =t_{q}=-\infty, \quad \text { если } \operatorname{Fix}\langle q\rangle=\varnothing .
\end{aligned}
$$

Определим еще одно подмножество группы $G \subseteq \mathrm{Homeo}_{+}(\mathbb{R})$ :

$$
C_{G}=\left(G \backslash G^{S}\right) \cup G_{\infty}^{S}
$$

Теорема 29 (Бекларян, 1996 г., [9], [14]). Пусть для группъ $G \subseteq \mathrm{Homeo}_{+}(\mathbb{R})$ не существует борелевской инвариантной меры, конечной на компактах. Для существования борелевской меры, конечной на компактах и проективно инвариантной относителъно группы $G$, необходимо и достаточно, чтобы одновременно выполнялисъ следующие условия:

1) множество $G_{\infty}^{S}$ является подгруппой и факторгруппа $G / G_{\infty}^{S}$ некоммутативна;

2) для всякого $g \in G^{S} \backslash C_{G}$ справедливы условия: $t_{g}, T_{g}$ конечны и для любых $t \in]-\infty, t_{g}[, T \in] T_{g},+\infty[$ выполнено соотношение

$$
\operatorname{sign}[g(t)-t]=-\operatorname{sign}[g(T)-T]
$$

3) для любых $g_{1}, g_{2} \in G^{S} \backslash C_{G}$ либо $t_{g_{1}}=t_{g_{2}}$ u $T_{g_{1}}=T_{g_{2}}$, либо $\left[t_{g_{1}}, T_{g_{1}}\right] \cap$ $\left[t_{g_{2}}, T_{g_{2}}\right]=\varnothing$.

Для групп, не имеющих инвариантной меры, мы можем сформулировать иной критерий существования проективно инвариантной меры, также использующий свойства подмножеств $G_{\infty}^{S}$ и $C_{G}$ исходной группы $G \subseteq \mathrm{Homeo}_{+}(\mathbb{R})$.

Теорема 30 (Бекларян, 1996 г., [10], [14]). Пусть для $G \subseteq \mathrm{Homeo}_{+}(\mathbb{R})$ не существует инвариантной борелевской меры, конечной на компактах. Для существования борелевской меры, конечной на компактах и проективно инвариантной относительно группы $G$, необходимо и достаточно, чтобы подмножества $G_{\infty}^{S}$ и $C_{G}$ являлисъ подгруппами и выполнялосъ условие $C_{G} \neq G_{\infty}^{S}$.

ЗАмечание 6 [10], [14]. Если для группы $G \subseteq$ Homeo+ $_{+}(\mathbb{R})$ существует борелевская мера $\mu$, конечная на компактах и проективно инвариантная относительно группы $G$, но не существует инвариантной борелевской меры, конечной на компактах, то имеют место следующие утверждения:

(a) $G_{\infty}^{S}=H_{G}$;

(b) подгруппы $G_{\infty}^{S}$ и $C_{G}$ являются нормальными, а факторгруппы $G / C_{G}$, $C_{G} / G_{\infty}^{S}$ являются коммутативными, причем факторгруппа $C_{G} / G_{\infty}^{S}-$ не циклическая;

(c) мера $\mu$ для подгруппы $C_{G}$ является инвариантной. 
Из замечания 1 следует, что подгруппа $C_{G}$ содержит свободно действующий элемент, а из замечания 6 следует, что для подгруппы $C_{G}$ существует инвариантная мера. Более того, из определения множества $C_{G}$ следует, что для подгруппы $C_{G}$ имеет место равенство

$$
C_{G}^{S}=G_{\infty}^{S}
$$

Поэтому, в силу замечания 6 , для факторгруппы $C_{G} / G_{\infty}^{S}$ справедливо равенство

$$
C_{G} / G_{\infty}^{S}=C_{G} / C_{G}^{S}
$$

Заметим, что критерий из теоремы 30 основан на свойствах подмножеств $C_{G}, C_{G}^{S}$. Сформулируем критерий, не использующий подмножества $C_{G}, C_{G}^{S}$, а основанный только лишь на наличии некоторой нормальной подгруппы со специальными свойствами.

Теорема 31 (Бекларян, 2002 г., [13], [14]). Пусть для $G \subseteq \mathrm{Homeo}_{+}(\mathbb{R})$ не существует инвариантной борелевской мерь, конечной на компактах. Для существования борелевской меры, конечной на компактах и проективно инвариантной относительно группы $G$, необходимо и достаточно, чтобъ существовала нормальная подгруппа $\Gamma \subseteq G$ с нециклической факторгруппой $\Gamma /\left\langle\Gamma^{S}\right\rangle$.

ЗАмечАниЕ 7. Из замечания 1 следует, что подгруппа Г содержит свободно действующий элемент, а из теоремы 22 следует, что для подгруппы Г существует инвариантная мера.

ЗАмЕчАниЕ 8. В силу предложения 3, замечания 2 и теоремы 30, для группы $G \subseteq$ Homeо $_{+}(\mathbb{R})$ из существования проективно инвариантной меры следует, что минимальное множество непусто.

Достаточное условие из теоремы 31 было сформулировано в работе [45] в иной, эквивалентной, форме. Формулировка была дана в терминах характера, построенного для группы $G$ с помощью меры, инвариантной относительно нормальной подгруппы Г.

Критерий существования проективно инвариантной меры в теореме 31 содержит трудно проверяемое условие существования нормальной подгруппы $Г$, для которой факторгруппа $\Gamma /\left\langle\Gamma^{S}\right\rangle$ не циклическая. Дадим формулировку критерия существования проективно инвариантной меры, в котором требование к нормальной подгруппе Г ослабляются и это компенсируется условием в терминах комбинаторных характеристик.

Tеорема 32 (Бекларян, 2002 г., [13], [14]). Пусть груnпа $G \subseteq \mathrm{Homeo}_{+}(\mathbb{R})$ содержит нормальную подгруппу $\Gamma$ с нетривиальной факторгруппой $\Gamma /\left\langle\Gamma^{S}\right\rangle$, что эквивалентно условию существования для подгруппы Г инвариантной мерь и свободно действующего элемента. Тогда либо факторгруппа $G / H_{G}$ содержит свободную подгруппу с двумя образующими, либо существует проективно инвариантная мера. 
В работе Планте (1983) [45] было сформулировано достаточное условие существования проективно инвариантной меры, которое является значительно более жестким, чем в теореме 32: для группы $G \subseteq$ Homeo+ $_{+}(\mathbb{R})$, содержащей нормальную подгруппу Г с инвариантной мерой и свободно действующим элементом, из аменабельности группы $G$ следует существование проективно инвариантной меры.

Мы можем вместо сильной альтернативы из теоремы 32 дать формулировку слабой альтернативы, но при этом факторгруппу заменить самой исходной группой. Такая слабая альтернатива для группы гомеоморфизмов прямой представляет собой как признак существования проективно инвариантной меры, так и признак существования свободной подгруппы с двумя образующими.

СЛЕДСТВИе 4. Пусть группа $G \subseteq \mathrm{Homeo}_{+}(\mathbb{R})$ содержит нормалъную подгруппу Г с нетривиальной факторгруппой $\Gamma /\left\langle\Gamma^{S}\right\rangle$, что эквивалентно условию существования для подгруппь Г инвариантной меры и свободно действующего элемента. Тогда или группа $G$ содержит свободную подгруппу с двумя образующими, или существует проективно инвариантная мера.

ДокАЗАТЕЛьство. Действительно, если группа $G$ не содержит свободную подгруппу с двумя образующими, то и факторгруппа $G / H_{G}$ не будет содержать свободную подгруппу с двумя образующими. Тогда по теореме 32 существует проективно инвариантная мера. Следствие доказано.

Дадим эквивалентную переформулировку теоремы 32, в которой все утверждения приводятся исключительно в терминах факторгруппы $G / H_{G}$.

Теорема 33 (Бекларян, 2002 г., [13], [14]). Пусть групnа $G \subseteq \mathrm{Homeo}_{+}(\mathbb{R})$ содержит нормальную подгруппу $\Gamma$ с нетривиальной факторгруппой $\Gamma /\left\langle\Gamma^{S}\right\rangle$, что эквивалентно условию существования для подгруппы Г инвариантной меры и свободно действующего элемента. Тогда факторгруппа $G / H_{G}$ либо содержит свободную подгруппу с двумя образующими, либо является разрешимой әруппой.

Тем не менее проверка наличия нормальной подгруппы $Г$ с инвариантной мерой и свободно действующим элементом также является сложной задачей. Хотелось бы оставить только лишь условие существования свободно действующего элемента. Оказывается, что это можно сделать. Ниже будет показано, что для группы со свободно действующим элементом критерием существования проективно инвариантной меры является отсутствие некоторой специальной подгруппы с двумя образующими.

Теорема 34 (Бекларян, 2013 г., [19]). Пусть $G \subseteq$ Homeo+ $_{+}(\mathbb{R})$ - груnпа со свободно действующим элементом $\bar{g} \in G(\mathrm{Fix} \bar{g}=\varnothing)$. Для существования проективно инвариантной меры необходимо и достаточно, чтобы в группе $G$ отсутствовала подгруппа $\Lambda \subseteq G, \Lambda=\langle p, q\rangle$, в которой элемент $p$ - свободно действуюший и элементы $p, q$ относительно точек $t_{0}, t_{1} \in E(G), t_{0}<t_{1}$, 
удовлетворяют следующим условиям:

$$
\begin{aligned}
p(t)>t, \quad t \in \mathbb{R}, & p\left(t_{0}\right) \in\left(t_{0}, t_{1}\right) ; \\
q\left(t_{0}\right)=t_{0}, \quad q\left(t_{1}\right)=t_{1}, & q(t)>t, \quad t \in\left(t_{0}, t_{1}\right) .
\end{aligned}
$$

Графики элементов $p, q$ имеют вид, указанный на рис. 2.

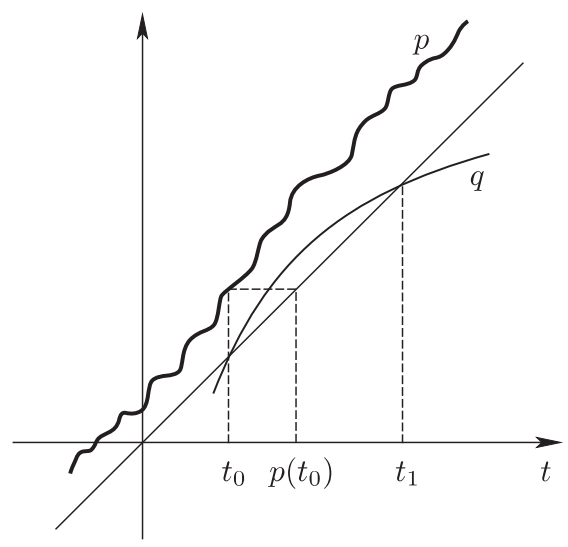

Рис. 2

$\mathrm{K}$ сожалению, условие отсутствия специальной подгруппы $\Lambda=\langle p, q\rangle$ не удается переформулировать в терминах комбинаторных характеристик. Вместе с тем такой критерий весьма удобен для изучения вопроса существования проективно инвариантной меры.

Критерии существования проективно инвариантной меры также могут быть переформулированы в терминах полусопряженности (см. определение 9).

Теорема 35 (Бекларян, 1996 г., [10], [14]). Пусть $G \subseteq$ Homeo+ $_{+}(\mathbb{R})$. Тогда следующие утверждения эквивалентны:

1) существует борелевская мера $\mu$, конечная на компактах и проективно-инвариантная относительно группь $G$;

2) группа $G$ полусопряжена некоторой группе ${ }_{*} G \subseteq \mathrm{Homeo}_{+}(\mathbb{R})$ афффинных преобразований прямой, сохраняющих ориентацию.

Связь между отображением $\eta$ и проективно инвариантной мерой $\mu$ из теоремы 35 описывается так же, как в замечании 5.

В терминах полусопряженности подгруппа $H_{G}$ (см. замечание $6: H_{G}=G_{\infty}^{S}$, если нет инвариантной меры) совпадает с ядром гомоморфизма $\eta^{\sharp}$, т. e. $\operatorname{ker} \eta^{\sharp}=$ $H_{G}$, а факторгруппа $G / H_{G}$ изоморфна группе аффинных преобразований прямой ${ }_{*} G$, т. е. ${ }_{*} G \simeq G / H_{G}$.

Несколько замечаний по истории вопроса. Вопрос существования проективно инвариантной меры для группы гомеоморфизмов прямой рассматривался в работе Планте [45]. В [45] все формулировки даются в терминах характера, 
построенного для группы $G$ с помощью меры, инвариантной относительно нормальной подгруппы Г. Вопросы, связанные с формулировкой критерия существования проективно инвариантной меры в терминах различных характеристик группы гомеоморфизмов прямой, а также описание носителя проективно инвариантной меры и эргодические свойства такой меры приводятся в обзоре автора [14].

\section{5. Цепочки вложений групп гомеоморфизмов прямой (окружности, интервала) и факторгрупп, а также соответствия классов таких групп и факторгрупп, порожденные различными их характеристиками}

Напомним, что $\mathbb{X}=\mathbb{R}, \mathbb{S}^{1}$. Через $\mathscr{W}(\mathbb{X})$ будем обозначать класс всевозможных групп $G \subseteq$ Homeо $_{+}(\mathbb{X})$, для которых минимальное множество непусто. Как отмечалось ранее, для любой группы $G \subseteq \mathrm{Homeo}_{+}\left(\mathbb{S}^{1}\right)$ минимальное множество непусто. Поэтому для окружности класс $\mathscr{W}\left(\mathbb{S}^{1}\right)$ совпадает с множеством всех подгрупп группы гомеоморфизмов $\mathrm{Homeo}_{+}\left(\mathbb{S}^{1}\right)$. Определим отображение

$$
\Psi: \mathscr{W}(\mathbb{X}) \mapsto \mathscr{W}, \quad \Psi(G)=G / H_{G}
$$

Лемма 2. Пусть $G \subseteq \mathscr{W}(\mathbb{X})$. Тогда фбакторгруппа $G / H_{G}$ также может быть реализована как группа гомеоморфизмов $\mathbb{X}$ с минимальным множеством, совпадающим с минимальным множеством исходной группы $G$.

ДокАзАтельство. Так как для любой группы $G \in \mathscr{W}(\mathbb{X})$ минимальное множество непусто, то либо оно является единственным и недискретным множеством $E(G)$, либо оно дискретно и объединение всевозможных минимальных множеств совпадает с замкнутым инвариантным множеством Fix $G^{S}$. Для любого элемента $\widehat{g} \in G / H_{G}$ через $g\left[H_{G}\right]$ будем обозначать соответствующий ему левый смежный класс группы $G$ по подгруппе $H_{G}$. Если минимальное множество не дискретное, то положим

$$
\widehat{g}(t)=g(t), \quad t \in E(G) .
$$

На дополнении $\mathbb{R} \backslash E(G)$, состоящем из не более чем счетного объединения связных максимальных открытых интервалов, продолжим отображение $\widehat{g}$ на каждом таком интервале в виде линейного растяжения так, чтобы оно задавало перекладывание этих интервалов. Такое отображение будет гомеоморфизмом. В случае, когда минимальное множество дискретное, проделываем то же самое с заменой множества $E(G)$ на $\operatorname{Fix} G^{S}$.

Очевидно, что при такой реализации факторгруппы $G / H_{G}$ ее минимальное множество совпадает с минимальным множеством исходной группы $G$. Лемма доказана.

СлЕДСТвИЕ 5. В силу леммы 2 мы можем считать, что отображение $\Psi$ действует из $\mathscr{W}(\mathbb{X})$ в себя, m.е.

$$
\Psi: \mathscr{W}(\mathbb{X}) \rightarrow \mathscr{W}(\mathbb{X})
$$


В разделе 1 мы определили классы групп, являющиеся подмножествами множества всех групп $\mathscr{W}$. Через

$$
\mathscr{K}(\mathbb{X}), \aleph(\mathbb{X}), \mathscr{N}_{P}(\mathbb{X}), \mathscr{S}(\mathbb{X}), \mathscr{E}(\mathbb{X}), \mathscr{A}(\mathbb{X}), \mathscr{N}(\mathbb{X})
$$

обозначим классы групп в множестве всех групп $\mathscr{W}(\mathbb{X})$ с одноименными названиями. В каждом из определенных выше классов групп, зададим следующие подклассы:

$$
\begin{aligned}
I \mathscr{K}(\mathbb{X}) & =\Psi(\mathscr{W}(\mathbb{X})) \cap \mathscr{K}(\mathbb{X}), & I \aleph(\mathbb{X}) & =\Psi(\mathscr{W}(\mathbb{X})) \cap \aleph(\mathbb{X}), \\
I \mathscr{N}_{P}(\mathbb{X}) & =\Psi(\mathscr{W}(\mathbb{X})) \cap \mathscr{N}_{P}(\mathbb{X}), & I \mathscr{S}(\mathbb{X}) & =\Psi(\mathscr{W}(\mathbb{X})) \cap \mathscr{S}(\mathbb{X}), \\
I \mathscr{E}(\mathbb{X}) & =\Psi(\mathscr{W}(\mathbb{X})) \cap \mathscr{E}(\mathbb{X}), & I \mathscr{A}(\mathbb{X}) & =\Psi(\mathscr{W}(\mathbb{X})) \cap \mathscr{A}(\mathbb{X}), \\
I \mathscr{N}(\mathbb{X}) & =\Psi(\mathscr{W}(\mathbb{X})) \cap \mathscr{N}(\mathbb{X}), & I \mathscr{W}(\mathbb{X}) & =\Psi(\mathscr{W}(\mathbb{X})) \cap \mathscr{W}(\mathbb{X})
\end{aligned}
$$

По сути класс групп с припиской буквы $I$ - это такой класс групп, элементы которых после операции факторизации $G / H_{G}$ попадают в отмеченный класс.

\section{1. Цепочки вложений факторгрупп и соответствия классов групп} гомеоморфизмов прямой (окружности), порожденные метрическими инвариантами. Будут описаны соответствия, порожденные инвариантной, проективно инвариантной, а также $\omega$-проективно инвариантной мерами.

а) Цепочки вложений факторгрупп и соответствия классов групп гомеоморфизмов прямой (окружности), порожденные инвариантной мерой. Описание таких соответствий классов групп можно получить, используя переформулировку эквивалентных друг другу теорем 25 и 26.

ПредлоЖениЕ 5. Справедливы следующие эквивалентные утверждения:

1) имеет место цепочка вложений для классов факторгрупп

$$
I \mathscr{K}(\mathbb{R})=I \mathscr{N}_{P}(\mathbb{R}) \subset I \mathscr{S}(\mathbb{R}) \subseteq I \mathscr{E}(\mathbb{R}) \subseteq I \mathscr{A}(\mathbb{R}) \subseteq I \mathscr{N}(\mathbb{R}) \subset I \mathscr{W}(\mathbb{R}) ;
$$

2) имеются соответствия классов групп, порожденные существованием (отсутствием) инвариантной мерь:

\begin{tabular}{|c|c|}
\hline существование инвариантной мерь & классъ групп \\
\hline+ & $\Psi^{-1}\left[I \mathscr{N}_{P}(\mathbb{R})\right]$ \\
\hline- & $\mathscr{W}(\mathbb{R}) \backslash \Psi^{-1}\left[I \mathscr{N}_{P}(\mathbb{R})\right]$ \\
\hline
\end{tabular}

ДокАЗАтельство. 1) Равенство

$$
I \mathscr{K}(\mathbb{R})=I \mathscr{N}_{P}(\mathbb{R})
$$

в точности совпадает с формулировкой теоремы 26. Все нестрогие вложения в (13) очевидны. Для аффинной группы $G=\langle t+1,2 t\rangle$ с двумя образующими минимальное множество совпадает со всей прямой, т. е. $E(G)=\mathbb{R}$. Поэтому $H_{G}=\langle e\rangle$ и, соответственно, $G=G / H_{G}$. Такая группа является разрешимой группой и для нее существует свободная подполугруппа с двумя образующими. Поэтому имеет место строгое вложение

$$
I \mathscr{N}_{P}(\mathbb{R}) \subset I \mathscr{S}(\mathbb{R}) .
$$


Более того, для такой группы не существует свободной подгруппы с двумя образующими, но, с другой стороны, существует свободная группа гомеоморфизмов $\mathbb{R}$ с минимальным множеством $E(G)=\mathbb{R}$. Поэтому также имеет место строгое вложение

$$
I \mathscr{N}(\mathbb{R}) \subset I \mathscr{W}(\mathbb{R}) .
$$

2) Приведенные соответствия классов групп в точности совпадают с формулировкой теоремы 25.

И наконец, эквивалентность утверждений 1) и 2) следует из эквивалентности утверждений теорем 25 и 26. Предложение доказано.

ЗАмЕЧАНИЕ 9. Имеет место вложение

$$
\mathscr{N}_{P}(\mathbb{R}) \subseteq \Psi^{-1}\left[I \mathscr{N}_{P}(\mathbb{R})\right]
$$

Действительно, если для группы $G$ не существует свободных подполугрупп с двумя образующими, т. е. $G \in \mathscr{N}_{P}(\mathbb{R})$, то и для факторгруппы $\Psi(G)=G / H_{G}$ не будет существовать свободных подполугрупп с двумя образующими, т. е. справедливо включение $\Psi(G) \in I \mathscr{N}_{P}(\mathbb{R})$, откуда и следует вложение.

В классе групп $G \subseteq$ Homeo+ $_{+}(\mathbb{R})$ гомеоморфизмов прямой с непустым минимальным множеством и со свойством $H_{G}=\langle e\rangle$ (группа совпадает со своей факторизацией) равенство $I \mathscr{K}(\mathbb{R})=I \mathscr{N}_{P}(\mathbb{R})$ из пункта 1$)$ предложения 5 можно сформулировать в форме критерия коммутативности.

СледСтвиЕ 6. Пусть $G \subseteq$ Homeo+ $_{+}(\mathbb{R})$, для группь $G$ существует непустое минимальное множество и справедливо равенство $H_{G}=\langle е\rangle . B$ этом случае группа $G$ является коммутативной тогда и только тогда, когда она не содержит свободных подполугрупп с двумя образующими.

ДокАЗАТЕЛЬСтво. Для такой группы выполнено условие $G=G / H_{G}$ и, соответственно, $G \in I \mathscr{W}(\mathbb{R})$. В таком случае утверждение следствия вытекает из равенства $I \mathscr{K}(\mathbb{R})=I \mathscr{N}_{P}(\mathbb{R})$ в пункте 1$)$ предложения 5. Следствие доказано.

Для окружности дадим переформулировку эквивалентных друг другу теорем 23 и 24.

ПредлОЖЕниЕ 6. Справедливы следующие эквивалентные утверждения:

1) имеет место цепочка вложений для классов факторгрупп

$$
I \mathscr{K}\left(\mathbb{S}^{1}\right)=I \mathscr{N}_{P}\left(\mathbb{S}^{1}\right)=I \mathscr{S}\left(\mathbb{S}^{1}\right)=I \mathscr{E}\left(\mathbb{S}^{1}\right)=I \mathscr{A}\left(\mathbb{S}^{1}\right)=I \mathscr{N}\left(\mathbb{S}^{1}\right) \subset I \mathscr{W}\left(\mathbb{S}^{1}\right)
$$

2) имеются соответствия классов групп, порожденные существованием (отсутствием) инвариантной меры:

\begin{tabular}{|c|c|}
\hline существование инвариантной мерь & классы групп \\
\hline+ & $\Psi^{-1}\left[I \mathscr{N}\left(\mathbb{S}^{1}\right)\right]$ \\
\hline- & $\mathscr{W}\left(\mathbb{S}^{1}\right) \backslash \Psi^{-1}\left[I \mathscr{N}\left(\mathbb{S}^{1}\right)\right]$ \\
\hline
\end{tabular}

ДоказАтельство. 1) Равенство

$$
I \mathscr{K}\left(\mathbb{S}^{1}\right)=I \mathscr{N}\left(\mathbb{S}^{1}\right)
$$


в точности совпадает с формулировкой теоремы 24 . В случае окружности $\mathbb{S}^{1}$ также существует свободная группа гомеоморфизмов $\mathbb{S}^{1}$ с двумя образующими и с минимальным множеством $E(G)=\mathbb{S}^{1}$. Следовательно,

$$
I \mathscr{N}\left(\mathbb{S}^{1}\right) \neq I \mathscr{W}\left(\mathbb{S}^{1}\right) .
$$

2) Приведенные соответствия в точности совпадают с формулировкой теоремы 23.

И наконец, эквивалентность утверждений 1) и 2) следует из эквивалентности утверждений теорем 23 и 24. Предложение доказано.

ЗАмечаниЕ 10. Имеет место вложение

$$
\mathscr{N}\left(\mathbb{S}^{1}\right) \subseteq \Psi^{-1}\left[I \mathscr{N}\left(\mathbb{S}^{1}\right)\right]
$$

Действительно, если для группы $G$ не существует свободных подгрупп с двумя образующими, т. е. $G \in \mathscr{N}\left(\mathbb{S}^{1}\right)$, то и для факторгруппы $\Psi(G)=G / H_{G}$ не будет существовать свободных подгрупп с двумя образующими, т. е. справедливо включение $\Psi(G) \in I \mathscr{N}\left(\mathbb{S}^{1}\right)$, откуда и следует вложение.

В классе групп $G \subseteq$ Нотео $_{+}\left(\mathbb{S}^{1}\right)$ гомеоморфизмов окружности со свойством $H_{G}=\langle e\rangle$ равенство $I \mathscr{K}\left(\mathbb{S}^{1}\right)=I \mathscr{N}\left(\mathbb{S}^{1}\right)$ из пункта 1) предложения 6 можно сформулировать в форме критерия коммутативности.

Следствие 7. Пусть $G \subseteq \mathrm{Homeo}_{+}\left(\mathbb{S}^{1}\right)$ и для группы $G$ справедливо равенство $H_{G}=\langle$ е (әруппа совпадает со своей факторизацией). В этом случае группа $G$ является коммутативной тогда и только тогда, когда она не содержит свободных подгрупп с двумя образующими.

ДокАзАтЕльство. Для такой группы выполнено условие $G=G / H_{G}$ и, соответственно, $G \in I \mathscr{W}\left(\mathbb{S}^{1}\right)$. В таком случае, утверждение следствия вытекает из равенства $I \mathscr{K}\left(\mathbb{S}^{1}\right)=I \mathscr{N}\left(\mathbb{S}^{1}\right)$ в пункте 1) предложения 6. Следствие доказано.

Из условия $E(G)=\mathbb{R}\left(E(G)=\mathbb{S}^{1}\right)$ следует условие $H_{G}=\langle e\rangle$, присутствующее в формулировке следствий 6 и 7. Это делает актуальным вопрос о наличии аналогов теоремы Данжуа об отсутствии минимального множества, изоморфного канторову множеству. Справедливость аналогов теоремы Данжуа следует ожидать в классе групп диффеоморфизмов окружности.

b) Цепочки вложений факторгрупп и соответствия классов групп гомеоморфизмов прямой (окружности), порожденные проективно инвариантной мерой. Описание такой шкалы соответствий классов групп можно получить используя переформулировку эквивалентных друг другу теорем 32 и 33.

Для этого через $\mathscr{W}(\mathbb{R} ; \Gamma)$ обозначим подкласс класса групп $\mathscr{W}(\mathbb{R})$, в котором для каждой группы существует некоторая нормальная подгруппа Г с инвариантной мерой и свободно действующим элементом. 
Определим более узкий подкласс групп следующим образом:

$$
\begin{aligned}
I \mathscr{K}(\mathbb{R} ; \Gamma) & =\Psi(\mathscr{W}(\mathbb{R} ; \Gamma)) \cap \mathscr{K}(\mathbb{R}), & & I \aleph(\mathbb{R} ; \Gamma)=\Psi(\mathscr{W}(\mathbb{R} ; \Gamma)) \cap \aleph(\mathbb{R}), \\
I \mathscr{N}_{P}(\mathbb{R} ; \Gamma) & =\Psi(\mathscr{W}(\mathbb{R} ; \Gamma)) \cap \mathscr{N}_{P}(\mathbb{R}), & & I \mathscr{S}(\mathbb{R} ; \Gamma)=\Psi(\mathscr{W}(\mathbb{R} ; \Gamma)) \cap \mathscr{S}(\mathbb{R}), \\
I \mathscr{E}(\mathbb{R} ; \Gamma) & =\Psi(\mathscr{W}(\mathbb{R} ; \Gamma)) \cap \mathscr{E}(\mathbb{R}), & & I \mathscr{A}(\mathbb{R} ; \Gamma)=\Psi(\mathscr{W}(\mathbb{R} ; \Gamma)) \cap \mathscr{A}(\mathbb{R}), \\
I \mathscr{N}(\mathbb{R} ; \Gamma) & =\Psi(\mathscr{W}(\mathbb{R} ; \Gamma)) \cap \mathscr{N}(\mathbb{R}), & I \mathscr{W}(\mathbb{R} ; \Gamma) & =\Psi(\mathscr{W}(\mathbb{R} ; \Gamma)) \cap \mathscr{W}(\mathbb{R}) .
\end{aligned}
$$

ПредложениЕ 7. Справедливы следующие эквивалентные утверждения:

1) имеет место иепочка вложений для классов факторгрупп

$$
\begin{aligned}
I \mathscr{K}(\mathbb{R} ; \Gamma) & =I \mathscr{N}_{P}(\mathbb{R} ; \Gamma) \subset I \mathscr{S}(\mathbb{R} ; \Gamma)=I \mathscr{E}(\mathbb{R} ; \Gamma)=I \mathscr{A}(\mathbb{R} ; \Gamma) \\
& =I \mathscr{N}(\mathbb{R} ; \Gamma) \subset I \mathscr{W}(\mathbb{R} ; \Gamma) ;
\end{aligned}
$$

2) имеются соответствия классов групп, порожденные существованием (отсутствием) проективно инвариантной мерь:

\begin{tabular}{|c|c|}
\hline $\begin{array}{c}\text { существование проективно } \\
\text { инвариантной мерьь }\end{array}$ & классы групп \\
\hline+ & $\Psi^{-1}[I \mathscr{N}(\mathbb{R} ; \Gamma)]$ \\
\hline- & $\Psi^{-1}[I \mathscr{W}(\mathbb{R} ; \Gamma) \backslash I \mathscr{N}(\mathbb{R} ; \Gamma)]$ \\
\hline
\end{tabular}

ДоказАтельство. 1) Цепочка

$$
I \mathscr{K}(\mathbb{R} ; \Gamma)=I \mathscr{N}_{P}(\mathbb{R} ; \Gamma) \subset I \mathscr{S}(\mathbb{R} ; \Gamma)
$$

непосредственно следует из соответствующей цепочки в пункте 1) предложения 5. Вторая цепочка равенств в (16) совпадает с формулировкой теоремы 33. В случае прямой $\mathbb{R}$ также существует группа гомеоморфизмов $\mathbb{R}$ с минимальным множеством $E(G)=\mathbb{R}$, свободной подгруппой с двумя образующими и нормальной подгруппой $\Gamma$ со свободным элементом и инвариантной мерой. Пример такой группы можно построить следующим образом. Образуем группу гомеоморфизмов прямой без инвариантной меры с минимальным множеством $E(G)=\mathbb{R}$ и элементами в виде накрытий гомеоморфизмов окружности. Так как $G=G / H_{G}$, то по теореме 24 для группы $G$ существует свободная подгруппа с двумя образующими. Рассмотрим группу

$$
\widehat{G}=\langle G, \bar{g}\rangle, \quad \bar{g}(t)=t+1 .
$$

Элемент $\bar{g}$ принадлежит центру группы $\widehat{G}$. Поэтому подгруппа $\Gamma=\langle\bar{g}\rangle$ группы $\widehat{G}$ является нормальной, для подгруппы $\Gamma$ существуют инвариантная мера и свободно действующий элемент $\bar{g}$. Очевидно, что для группы $\widehat{G}$ также существует свободная подгруппа с двумя образующими. Следовательно,

$$
I \mathscr{N}(\mathbb{R} ; \Gamma) \neq I \mathscr{W}(\mathbb{R} ; \Gamma) .
$$

2) Приведенные соответствия в точности совпадают с формулировкой теоремы 32 .

И наконец, эквивалентность утверждений 1) и 2) следует из эквивалентности утверждений теорем 32 и 33. Предложение доказано. 
ЗАмечаниЕ 11. Имеет место вложение

$$
\mathscr{N}(\mathbb{R} ; \Gamma) \subseteq \Psi^{-1}[I \mathscr{N}(\mathbb{R} ; \Gamma)]
$$

Действительно, если для группы $G$ не существует свободных подгрупп с двумя образующими, т. е. $G \in \mathscr{N}(\mathbb{R} ; \Gamma)$, то и для факторгруппы $\Psi(G)=G / H_{G}$ не будет существовать свободных подгрупп с двумя образующими, т. е. справедливо включение $\Psi(G) \in I \mathscr{N}(\mathbb{R} ; \Gamma)$, откуда и следует вложение.

В классе групп $G \subseteq$ Homeo+ $_{+}(\mathbb{R})$ гомеоморфизмов прямой со свойством $H_{G}=\langle e\rangle$ равенство $I \mathscr{S}(\mathbb{R} ; \Gamma)=I \mathscr{N}(\mathbb{R} ; \Gamma)$ из пункта 1$)$ предложения 7 можно сформулировать в форме критерия разрешимости.

СледСтвие 8. Пусть $G \subseteq$ Нотео $_{+}(\mathbb{R})$, существует нормальная подгруппа Г со свободно действующим элементом и инвариантной мерой и, кроме того, для группы $G$ справедливо равенство $H_{G}=\langle$ р) (группа совпадает со своей факторизацией). В этом случае группа $G$ является разрешимой группой тогда и только тогда, когда она не содержит свободных подгрупп с двумя образующими.

ДокАЗАтЕльство. Для такой группы выполнено условие $G=G / H_{G}$ и, соответственно, $G \in I \mathscr{W}(\mathbb{R} ; \Gamma)$. В таком случае утверждение следствия вытекает из равенства $I \mathscr{S}(\mathbb{R} ; \Gamma)=I \mathscr{N}(\mathbb{R} ; \Gamma)$ из пункта 1$)$ предложения 7 . Следствие доказано.

с) Соответствия классов групп гомеоморфизмов прямой (окружности), порожденные серией метрических инвариантов. Используя соответствия из предыдущих разделов, мы можем сформировать сводные соответствия, порожденные инвариантной, проективно инвариантной, а также $\omega$-проективно инвариантной мерами.

В случае прямой $\mathbb{R}$ используем соответствия классов групп из предложений 5 и 7.

ПРЕДЛОЖЕНИЕ 8. Существование (отсутствие) инвариантной, проективно инвариантной и шкалу соответствий классов групп:

\begin{tabular}{|c|c|c|c|}
\hline $\begin{array}{c}\text { существование } \\
\text { инвариантной } \\
\text { мерь }\end{array}$ & $\begin{array}{c}\text { существование } \\
\text { проективно } \\
\text { инвариантной } \\
\text { мерьь }\end{array}$ & $\begin{array}{c}\text { существование } \\
\text { ш-проективно } \\
\text { инвариантной } \\
\text { мерь }\end{array}$ & $\begin{array}{c}\text { классы } \\
\text { групn }\end{array}$ \\
\hline+ & + & + & $\Psi^{-1}\left[I \mathscr{N}_{P}(\mathbb{R})\right]$ \\
\hline- & + & + & $\Psi^{-1}\left[I \mathscr{N}(\mathbb{R} ; \Gamma) \backslash I \mathscr{N}_{P}(\mathbb{R})\right]$ \\
\hline- & - & + & $\begin{array}{c}\mathscr{W}(\mathbb{R}) \backslash \Psi^{-1}[I \mathscr{N}(\mathbb{R} ; \Gamma) \cup \\
\left.I \mathscr{N}_{P}(\mathbb{R})\right]\end{array}$ \\
\hline
\end{tabular}


ДокАЗАтЕЛьство. Прокомментировать следует только третью строку. По теореме 31 для группы $G$ без инвариантной меры, но с проективно инвариантной мерой существует нормальная подгруппа Г с инвариантной мерой и свободно действующим элементом. Следовательно, для любой группы из $\Psi^{-1}[I \mathscr{W}(\mathbb{R}) \backslash$ $\left.\left\{I \mathscr{N}(\mathbb{R} ; \Gamma) \cup I \mathscr{N}_{P}(\mathbb{R})\right\}\right]$ не существует ни инвариантной, ни проективно инвариантной меры, но существует $\omega$-проективно инвариантная мера (теорема 16). Остается заметить, что

$$
\Psi^{-1}\left[I \mathscr{W}(\mathbb{R}) \backslash\left\{I \mathscr{N}(\mathbb{R} ; \Gamma) \cup I \mathscr{N}_{P}(\mathbb{R})\right\}\right]=\mathscr{W}(\mathbb{R}) \backslash \Psi^{-1}\left[I \mathscr{N}(\mathbb{R} ; \Gamma) \cup I \mathscr{N}_{P}(\mathbb{R})\right]
$$

Предложение доказано.

Используя соответствия из предложения 6, можем сформировать сводные соответствия классов групп в случае окружности $\mathbb{S}^{1}$. Напомним, что в случае окружности $\mathbb{S}^{1}$ всякая проективно инвариантная мера является инвариантной.

ПРеДЛОЖЕНИЕ 9. Существование (отсутствие) инвариантной и $\omega$-проективно инвариантной мер порождает следующую шкалу соответствий класcов групn:

\begin{tabular}{|c|c|c|}
\hline $\begin{array}{c}\text { существование } \\
\text { инвариантной } \\
\text { мерьь }\end{array}$ & $\begin{array}{c}\text { существование } \\
\omega \text {-проективно } \\
\text { инвариантной мерьь }\end{array}$ & $\begin{array}{c}\text { классъь } \\
\text { групп }\end{array}$ \\
\hline+ & + & $\Psi^{-1}\left[I \mathscr{N}\left(\mathbb{S}^{1}\right)\right]$ \\
\hline- & + & $\mathscr{W}\left(\mathbb{S}^{1}\right) \backslash \Psi^{-1}\left[I \mathscr{N}\left(\mathbb{S}^{1}\right)\right]$ \\
\hline
\end{tabular}

ДокАзАтельство. Такие соответствия следуют из соответствий предложения 6 , теоремы 16 о существовании $\omega$-проективно инвариантной меры и того факта, что в случае окружности всякая проективно инвариантная мера является инвариантной. Предложение доказано.

5.2. Цепочки вложений групп гомеоморфизмов прямой (окружности, интервала) и факторгрупп, а также соответствия классов таких групп и факторгрупп, порожденные их ростом. Далее через

$$
I \mathscr{K}_{f}(\mathbb{X}), I \aleph_{f}(\mathbb{X}), I \mathscr{N}_{P f}(\mathbb{X}), I \mathscr{S}_{f}(\mathbb{X}), I \mathscr{E}_{f}(\mathbb{X}), I \mathscr{A}_{f}(\mathbb{X}), I \mathscr{N}_{f}(\mathbb{X}), I \mathscr{W}_{f}(\mathbb{X})
$$

будем обозначать подклассы конечно порожденных групп из классов групп

$$
I \mathscr{K}(\mathbb{X}), I \aleph(\mathbb{X}), I \mathscr{N}_{P}(\mathbb{X}), I \mathscr{S}(\mathbb{X}), I \mathscr{E}(\mathbb{X}), I \mathscr{A}(\mathbb{X}), I \mathscr{N}(\mathbb{X}), I \mathscr{W}(\mathbb{X})
$$

соответственно.

а) Цепочки вложений для факторгрупп и соответствия классов факторгрупп, порожденные их ростом. Заметим, что факторгруппа конечно порожденной группы является конечно порожденной и для конечно порожденной группы $G \subseteq \mathrm{Homeo}_{+}(\mathbb{X})$ минимальное множество всегда непусто. В таком случае для всякой конечно порожденной группы $G \subseteq \mathrm{Homeo}_{+}(\mathbb{X})$ будут выполняться включения

$$
G \in \mathscr{W}_{f}(\mathbb{X}), \quad G / H_{G} \in I \mathscr{W}_{f}(\mathbb{X}),
$$


а также оценка для роста группы

$$
\lambda\left(G / H_{G}\right) \leqslant \lambda(G) .
$$

Для класса конечно порожденных групп вложения (13) могут быть уточнены.

Лемма 3. Имеет место иепочка равенств для классов конечно порожденнъих факторгрупn

$$
I \mathscr{K}_{f}(\mathbb{X})=I \aleph_{f}(\mathbb{X})=I \mathscr{N}_{P f}(\mathbb{X})
$$

ДокАЗАТЕЛЬСтво. Если мы покажем, что для класса конечно порожденных нильпотентных групп $I \aleph_{f}(\mathbb{X})$ справедливо вложение $I \aleph_{f}(\mathbb{X}) \subseteq I \mathscr{N}_{P f}(\mathbb{X})$, то из равенства $I \mathscr{K}_{f}(\mathbb{X})=I \mathscr{N}_{P f}(\mathbb{X})$ (см. вложения $\left.(13),(14)\right)$ и будет следовать доказательство леммы. Докажем вложение. Действительно, если вложение не выполняется, то найдется конечно порожденная нильпотентная группа из $I \aleph_{f}(\mathbb{X})$ со свободной подполугруппой с двумя образующими. Но для такой группы рост экспоненциальный, что противоречит теореме Громова (теорема 2). Лемма доказана.

Опишем соответствия классов факторгрупп, порожденные их ростом.

ПредлОжЕниЕ 10. Справедливы следующие два утверждения:

1) имеет место цепочка вложений для классов конечно порожденных факmopгpynn

$$
\begin{aligned}
I \mathscr{K}_{f}(\mathbb{R}) & =I \aleph_{f}(\mathbb{R})=I \mathscr{N}_{P f}(\mathbb{R}) \subset I \mathscr{S}_{f}(\mathbb{R}) \subseteq I \mathscr{E}_{f}(\mathbb{R}) \\
& \subseteq I \mathscr{A}_{f}(\mathbb{R}) \subseteq I \mathscr{N}(\mathbb{R}) \subset I \mathscr{W}_{f}(\mathbb{R}) ;
\end{aligned}
$$

2) имеются соответствия классов конечно порожденных факторгрупп, порожденные ростом факторгруппы:

\begin{tabular}{|c|c|}
\hline рост факторгруппъ $\lambda\left(G / H_{G}\right)$ & классъ факторгрупп \\
\hline полиномиальный & $I \mathscr{N}_{P}(\mathbb{R})$ \\
\hline экспоненциалъный & $I_{\mathscr{W}}(\mathbb{R}) \backslash I \mathscr{N}_{P f}(\mathbb{R})$ \\
\hline
\end{tabular}

ДокАЗАтЕльство. 1) Вложения (19) являются следствием вложений (13) и леммы 3 .

2) Приведенные соответствия непосредственно следуют из равенств

$$
I \mathscr{K}_{f}(\mathbb{R})=I \aleph_{f}(\mathbb{R})=I \mathscr{N}_{P f}(\mathbb{R})
$$

и того, что для группы со свободной подполугруппой с двумя образующими рост экспоненциальный.

Предложение доказано.

Как видно из полученных соответствий, рост факторгрупп $G / H_{G}$ групп гомеоморфизмов прямой $G \subseteq \mathrm{Homeo}_{+}(\mathbb{R})$ также обладает экстремалъным свойством: он либо полиномиальный, либо экспоненциальный. 
ЗАмечание 12. Имеет место вложение

$$
\mathscr{N}_{P f}(\mathbb{R}) \subseteq \Psi^{-1}\left[I \mathscr{N}_{P f}(\mathbb{R})\right] .
$$

Действительно, если для группы $G$ не существует свободных подполугрупп с двумя образующими, т. е. $G \in \mathscr{N}_{P f}(\mathbb{R})$, то и для факторгруппы $\Psi(G)=G / H_{G}$ не будет существовать свободных подполугрупп с двумя образующими, т. е. справедливо включение $\Psi(G) \in I \mathscr{N}_{P f}(\mathbb{R})$, откуда и следует вложение.

В классе конечно порожденных групп $G \subseteq \mathrm{Homeo}_{+}(\mathbb{R})$ гомеоморфизмов прямой со свойством $H_{G}=\langle e\rangle$ равенство $I \mathscr{K}_{f}(\mathbb{R})=I \mathscr{N}_{P f}(\mathbb{R})$ из пункта 1$)$ предложения 10 можно сформулировать в форме критерия полиномиальности роста группы.

СледСтвиЕ 9. Пусть $G \subseteq \mathrm{Homeo+}_{+}(\mathbb{R})$ - конечно порожденная группа, для которой справедливо равенство $H_{G}=\langle$ е (группа совпадает со своей факторизацией). В этом случае группа $G$ имеет полиномиальный рост тогда и только тогда, когда она не содержит свободных подполугрупп с двумя образующими.

ДокАзАтЕЛЬство. Для такой группы имеет место равенство $G=G / H_{G}$ и, соответственно, $G \in I_{\mathscr{W}}(\mathbb{R})$. В таком случае утверждение следствия вытекает из равенства $I \mathscr{K}_{f}(\mathbb{R})=I \mathscr{N}_{P f}(\mathbb{R})$. Следствие доказано.

Сформулируем два полезных свойства для групп промежуточного роста.

Следствие 10. Пусть $G \subseteq \operatorname{Homeo}_{+}(\mathbb{R})$ - конечно порожденная группа с промежуточным ростом. Тогда для такой группы справедливо условие $H_{G} \neq\langle$ $\left.\rangle\right\rangle$ (группа не совпадает со своей факторизацией) и существует инвариантная мера.

ДокАзАтельство. Утверждение $H_{G} \neq\langle e\rangle$ докажем от противного. Пусть $H_{G}=\langle e\rangle$. Тогда $G=G / H_{G}$ и, соответственно, $G \in I_{f}(\mathbb{R})$. В таком случае рост $\lambda(G)$ должен быть либо полиномиальным, либо экспоненциальным. Противоречие. Следовательно, должно выполняться условие $H_{G} \neq\langle e\rangle$.

Для группы промежуточного роста должно выполняться условие

$$
G \in \mathscr{N}_{P f}(\mathbb{R}) .
$$

По замечанию 12 для такой группы справедливо включение $G \in \Psi^{-1}\left[\operatorname{IN}_{P f}(\mathbb{R})\right]$. Тогда из соответствий классов групп в предложении 5 следует, что для такой группы существует инвариантная мера.

Следствие доказано.

Для классов групп гомеоморфизмов окружности полученные результаты могут быть усилены.

ПредлОЖенИЕ 11. Справедливы следующие два утверждения:

1) имеет место цепочка вложений для классов конечно порожденных факmopгpynn

$$
\begin{aligned}
I \mathscr{K}_{f}\left(\mathbb{S}^{1}\right) & =I \aleph_{f}\left(\mathbb{S}^{1}\right)=I \mathscr{N}_{P f}\left(\mathbb{S}^{1}\right)=I \mathscr{S}_{f}\left(\mathbb{S}^{1}\right)=I \mathscr{E}_{f}\left(\mathbb{S}^{1}\right) \\
& =I \mathscr{A}_{f}\left(\mathbb{S}^{1}\right)=I \mathscr{N}_{f}\left(\mathbb{S}^{1}\right) \subset I \mathscr{W}_{f}\left(\mathbb{S}^{1}\right) ;
\end{aligned}
$$


2) имеются соответствия классов конечно порожденных факторгрупп, порожденные ростом факторгруппь:

\begin{tabular}{|c|c|}
\hline рост факторгруппы $\lambda\left(G / H_{G}\right)$ & классы групп \\
\hline полиномиальный & $I \mathscr{N}_{f}\left(\mathbb{S}^{1}\right)$ \\
\hline экспоненциалъный & $I_{f}\left(\mathbb{S}^{1}\right) \backslash I \mathscr{N}_{f}\left(\mathbb{S}^{1}\right)$ \\
\hline
\end{tabular}

ДоказАтельство. 1) Вложения (20) являются следствием вложений (14) и леммы 3.

2) Приведенные соответствия непосредственно следуют из вложений (20) и того, что для группы со свободной подполугруппой (тем более группой) с двумя образующими рост экспоненциальный.

Предложение доказано.

Как видно из полученных соответствий, рост факторгрупп $G / H_{G}$ групп гомеоморфизмов окружности $G \subseteq \mathrm{Homeo}_{+}\left(\mathbb{S}^{1}\right)$ также обладает экстремалъным свойством: он либо полиномиальный, либо экспоненциальный.

ЗАмечАНИЕ 13. Имеет место вложение

$$
\mathscr{N}_{f}\left(\mathbb{S}^{1}\right) \subseteq \Psi^{-1}\left[I \mathscr{N}_{f}\left(\mathbb{S}^{1}\right)\right]
$$

Действительно, если для группы $G$ не существует свободных подгрупп с двумя образующими, т. е. $G \in N_{f}\left(\mathbb{S}^{1}\right)$, то и для факторгруппы $\Psi(G)=G / H_{G}$ не будет существовать свободных подгрупп с двумя образующими, т. е. справедливо включение $\Psi(G) \in I \mathscr{N}_{f}\left(\mathbb{S}^{1}\right)$, откуда и следует вложение.

В классе групп $G \subseteq$ Homeо $_{+}\left(\mathbb{S}^{1}\right)$ гомеоморфизмов прямой со свойством $H_{G}=\langle e\rangle$ равенство $I \mathscr{K}_{f}\left(\mathbb{S}^{1}\right)=I \mathscr{N}_{f}\left(\mathbb{S}^{1}\right)$ из пункта 1) предложения 11 можно сформулировать в форме критерия полиномиальности роста группы.

СлЕДСТвиЕ 11. Пусть $G \subseteq \mathrm{Homeo}_{+}\left(\mathbb{S}^{1}\right)$ - конечно порожденная группа, для которой справедливо равенство $H_{G}=\langle е\rangle$ (группа совпадает со своей факторизацией). В этом случае группа $G$ имеет полиномиальный рост тогда и только тогда, когда она не содержит свободных подгрупп с двумя образующими.

ДокАЗАТЕЛЬСтво. Из условия $E(G)=\mathbb{S}^{1}$ следует, что $H_{G}=\langle e\rangle$. Поэтому $G=G / H_{G}$ и, соответственно, $G \in I_{f}\left(\mathbb{S}^{1}\right)$. В таком случае утверждение следствия вытекает из равенства $I \mathscr{K}_{f}\left(\mathbb{S}^{1}\right)=I \mathscr{N}_{f}\left(\mathbb{S}^{1}\right)$. Следствие доказано.

Сформулируем два полезных свойства относительно групп промежуточного роста.

СлеДСтвиЕ 12. Пусть $G \subseteq$ Homeо $_{+}\left(\mathbb{S}^{1}\right)-$ конечно порожденная группа с промежуточным ростом. Тогда для такой группы выполнено условие $H_{G} \neq\langle$ ер (әруппа не совпадает со своей факторизащией) и существует инвариантная мера. 
ДокАзАтельство. Утверждение $H_{G} \neq\langle e\rangle$ докажем от противного. Пусть $H_{G}=\langle e\rangle$. Тогда $G=G / H_{G}$ и, соответственно, $G \in I_{f}\left(\mathbb{S}^{1}\right)$. В таком случае рост $\lambda(G)$ должен быть либо полиномиальным, либо экспоненциальным. Противоречие. Следовательно, должно выполняться условие $H_{G} \neq\langle e\rangle$.

Для группы промежуточного роста должно выполняться условие

$$
G \in \mathscr{N}_{P f}(\mathbb{S})
$$

По замечанию 13 для такой группы справедливо включение $G \in \Psi^{-1}\left[I \mathscr{N}_{P f}(\mathbb{S})\right]$. Тогда из соответствий классов групп в предложении 6 следует, что для такой группы существует инвариантная мера.

Следствие доказано.

b) Цепочки вложений групп диффеоморфизмов интервала, окружности и соответствия классов таких групп, порожденные их ростом. Мы определим один важный класс конечно порожденных групп диффеоморфизмов окружности и докажем его “массивность", а также сформулируем критерий нильпотентности для групп из такого класса.

ОПредЕлЕНиЕ 11. Диффеоморфизмы $g_{1}, g_{2} \in \operatorname{Diff}^{1}\left(\mathbb{S}^{1}\right), g_{1} \neq g_{2}$, называются взаимно трансверсальными, если из условия $g_{1}(t)=g_{2}(t), t \in \mathbb{S}^{1}$, следует, что

$$
\dot{g}_{1}(t) \neq \dot{g}_{2}(t) .
$$

Важное замечание состоит в том, что если $G$ - группа с образующими $g_{j} \in$ $\operatorname{Diff}^{1}\left(\mathbb{S}^{1}\right), j=1, \ldots, s$, и взаимно трансверсальными элементами, то для любого $t \in \mathbb{S}^{1}$, за исключением счетного множества точек, группа $G$ действует свободно на орбите $G(t)$.

Важно знать, как много групп с такими свойствами? Ответ на этот вопрос дает следующая теорема.

Теорема 36 (Бекларян, 2012 г., [17]). Множество свободных групп

$$
G=\left\langle g_{1}, \ldots, g_{s}\right\rangle, \quad g_{j} \in \operatorname{Diff}^{2}\left(\mathbb{S}^{1}\right), \quad j=1, \ldots, s,
$$

диффеоморфизмов окружности с фиксированным числом s образующих, для которых элементы взаимно трансверсальны, является в метрике пространства $\bigotimes_{s} \operatorname{Diff}^{2}\left(\mathbb{S}^{1}\right)$ счетным пересечением открытых всюоу плотных подмножеств (массивным множеством).

Ранее в теореме 24 была установлена альтернатива для факторгруппы $G / H_{G}$. Можно ли для группы диффеоморфизмов гладкости два с взаимно трансверсальными элементами получить дополнительные сведения о свойствах исходной группы $G$ ? В действительности, для рассматриваемого класса групп имеет место теорема Данжуа об отсутствии минимального множества, гомеоморфного канторову множеству, и положительный ответ на поставленный вопрос дается следующей теоремой. 
Теорема 37 (Бекларян, 2012 г., [16]). Пусть груnпа $G=\left\langle g_{1}, \ldots, g_{s}\right\rangle$ являтся группой диффеоморфизмов с элементами из $\operatorname{Diff}^{2}\left(\mathbb{S}^{1}\right)$, которье являются взаимно трансверсальными. Тогда либо факторгруппа $G / H_{G}$ содержит свободную подгруппу с двумя образующими, либо группа $G$ является почти нильпотентной.

Заметим, что теорема 37 допускает следующую переформулировку, не использующую подгруппу $H_{G}$.

TеOрема 38. Пусть группа $G=\left\langle g_{1}, \ldots, g_{s}\right\rangle$ является группой диффеоморфизмов с элементами из $\operatorname{Diff}^{2}\left(\mathbb{S}^{1}\right)$, которые являются взаимно трансверсальными. Тогда либо группа $G$ содержит свободную подгруппу с двумя образующими, либо группа $G$ является почти нильпотентной.

ДокАзАтельство. Достаточно показать, что из существования свободной подгруппы с двумя образующими в факторгруппе $G / H_{G}$ следует существование свободной подгруппы с двумя образующими в группе $G$ и наоборот. Действительно, если в факторгруппе $G / H_{G}$ существует свободная подгруппа с двумя образующими, то очевидно, что свободная подгруппа с двумя образующими будет существовать и в исходной группе $G$. Обратно, если в группе $G$ существует свободная подгруппа с двумя образующими, то в силу теоремы 37 свободная подгруппа с двумя образующими будет существовать и в факторгруппе $G / H_{G}$. Теорема доказана.

В дальнейшем для обозначения классов подгрупп группы гомеоморфизмов $\mathrm{Homeo}_{+}([0,1])$, групп диффеоморфизмов той или иной гладкости и групп диффеоморфизмов с взаимно трансверсальными элементами будем также использовать обозначения (12), (15), (17). Для этого следует соответственно вместо $\mathbb{X}$ подставить $[0,1]$, указать в виде индекса класс гладкости, обозначить условие трансверсальности значком $\perp$. Например, $\mathscr{W}([0,1]), \mathscr{W}^{1+\alpha}(\mathbb{X}), \stackrel{\mathscr{W}}{ }(\mathbb{X})$.

Приведем соответствия классов групп диффеоморфизмов, основанные на теореме 38 .

ПредлоЖениЕ 12. Справедливы следующие два утверждения:

1) имеет место чепочка вложений для классов конечно порожденных групп диффеоморфизмов

$$
\begin{aligned}
\perp \mathscr{K}_{f}^{2}\left(\mathbb{S}^{1}\right) & \subset\left[{ }^{\perp} \mathscr{N}_{P f}^{2}\left(\mathbb{S}^{1}\right) \cap{ }^{\perp} \mathscr{S}_{f}^{2}\left(\mathbb{S}^{1}\right)\right] \subseteq{ }^{\perp} \widehat{\aleph}_{f}^{2}\left(\mathbb{S}^{1}\right)=\perp \widehat{\mathscr{S}}_{f}^{2}\left(\mathbb{S}^{1}\right)={ }^{\perp} \mathscr{E}_{f}^{2}\left(\mathbb{S}^{1}\right) \\
& ={ }^{\perp} \mathscr{A}_{f}^{2}\left(\mathbb{S}^{1}\right)={ }^{\perp} \mathscr{N}_{f}^{2}\left(\mathbb{S}^{1}\right)={ }^{\perp} \mathscr{N}_{P f}^{2}\left(\mathbb{S}^{1}\right) \subset{ }^{\perp} \mathscr{W}_{f}^{2}\left(\mathbb{S}^{1}\right)
\end{aligned}
$$

2) имеются соответствия классов конечно порожденных групп дифбеоморфизмов, порожденные ростом группы:

\begin{tabular}{|c|c|}
\hline рост группъ $\lambda(G)$ & классъ групп \\
\hline полиномиалъный & $\perp \mathscr{N}_{f}^{2}\left(\mathbb{S}^{1}\right)$ \\
\hline экспоненциалъный & $\perp \mathscr{W}_{f}^{2}\left(\mathbb{S}^{1}\right) \backslash \perp \mathscr{N}_{f}^{2}\left(\mathbb{S}^{1}\right)$ \\
\hline
\end{tabular}


ДокАзАтельство. 1) Первое вложение в цепочке

$$
\perp \mathscr{K}_{f}^{2}\left(\mathbb{S}^{1}\right) \subset\left[{ }^{\perp} \mathscr{N}_{P f}^{2}\left(\mathbb{S}^{1}\right) \cap{ }^{\perp} \mathscr{S}_{f}^{2}\left(\mathbb{S}^{1}\right)\right] \subseteq{ }^{\perp} \widehat{\aleph}_{f}^{2}\left(\mathbb{S}^{1}\right)
$$

очевидно, а второе является следствием теоремы Розенблатта (теоремы 3). Цепочка равенств

$$
{ }^{\perp} \widehat{\aleph}_{f}^{2}\left(\mathbb{S}^{1}\right)=\perp \widehat{\mathscr{S}}_{f}^{2}\left(\mathbb{S}^{1}\right)={ }^{\perp} \mathscr{E}_{f}^{2}\left(\mathbb{S}^{1}\right)={ }^{\perp} \mathscr{A}_{f}^{2}\left(\mathbb{S}^{1}\right)={ }^{\perp} \mathscr{N}_{f}^{2}\left(\mathbb{S}^{1}\right)
$$

совпадает с утверждением теоремы 38. Равенство ${ }^{\perp} \mathscr{N}_{f}^{2}\left(\mathbb{S}^{1}\right)={ }^{\perp} \mathscr{N}_{P f}^{2}\left(\mathbb{S}^{1}\right)$ следует из предыдущей цепочки равенств и справедливости вложения ${ }^{\perp} \mathscr{N}_{P f}^{2}\left(\mathbb{S}^{1}\right) \subseteq$ $\perp \mathscr{N}_{f}^{2}\left(\mathbb{S}^{1}\right)$. Последнее вложение в $(21)$ следует из теоремы 36.

2) Приведенные соответствия непосредственно следуют из вложений (21), того факта, что группа со свободной погруппой с двумя образующими имеет экспоненциальный рост, а также теоремы Громова (теоремы 2) об эквивалентности условия полиномиальности роста группы условию почти нильпотентности.

Предложение доказано.

Как видно из приведенных соответствий, рост групп диффеоморфизмов $G \subseteq \operatorname{Diff}^{2}\left(\mathbb{S}^{1}\right)$ с взаимно трансверсальными элементами также обладает экстремальным свойством: он либо полиномиальный, либо экспоненциальный.

Приведем соответствия классов групп диффеоморфизмов, основанные на теореме Наваса (теорема 11).

ПРЕДЛОЖЕНИЕ 13. При любом $\alpha>0$ справедливы следующие два утверждения:

1) имеет место иепочка вложений для классов конечно порожденнъх групп дифбеоморфизмов

$$
\begin{aligned}
\mathscr{K}_{f}^{1+\alpha}([0,1]) & \subset \widehat{\aleph}_{f}^{1+\alpha}([0,1])=\mathscr{N}_{P f}^{1+\alpha}([0,1]) \subseteq \widehat{\mathscr{S}}_{f}^{1+\alpha}([0,1]) \\
& \subseteq \mathscr{E}_{f}^{1+\alpha}([0,1]) \subseteq \mathscr{A}_{f}^{1+\alpha}([0,1]) \subseteq \mathscr{N}_{f}^{1+\alpha}([0,1]) \subset \mathscr{W}_{f}^{1+\alpha}([0,1])
\end{aligned}
$$

2) имеются соответствия классов конечно порожденных групп дифбеоморфизмов, порожденные ростом группы:

\begin{tabular}{|c|c|}
\hline рост группы $\lambda(G)$ & классы групп \\
\hline полиномиальный & $\mathscr{N}_{P f}^{1+\alpha}([0,1])$ \\
\hline экспоненциалъный & $\mathscr{W}_{f}^{1+\alpha}([0,1]) \backslash \mathscr{N}_{P f}^{1+\alpha}([0,1])$ \\
\hline
\end{tabular}

ДокАЗАтЕльство. 1) Первое вложение в цепочке

$$
\mathscr{K}_{f}^{1+\alpha}([0,1]) \subset \widehat{\aleph}_{f}^{1+\alpha}([0,1])=\mathscr{N}_{P f}^{1+\alpha}([0,1])
$$

очевидно. Равенство является следствием теорем Громова (теоремы 2) и Наваса (теорема 11). Действительно, по теореме Громова всякая конечно порожденная почти нильпотентная группа имеет полиномиальный рост и поэтому является группой без свободных подполугрупп с двумя образующими. С другой 
стороны, по теореме Наваса всякая конечно порожденная группа без свободных подполугрупп с двумя образующими является нильпотентной. Остальные вложения в цепочке (24) очевидны.

2) Приведенные соответствия в точности совпадают с утверждениями теорем Наваса (теорема 11) и Громова (теоремы 2) и следствия 1.

Предложение доказано.

Как видно из приведенных соответствий, рост групп диффеоморфизмов $\mathscr{G} \subseteq \operatorname{Diff}^{1+\alpha}([0,1])$ также обладает экстремалъным свойством: он либо полиномиальный, либо экспоненциальный. На этой основе можем сформулировать простое следствие.

СЛЕДСТВИЕ 13. В классе конечно порожденных групп дифбеоморфизмов $G \subseteq \operatorname{Diff}^{2}\left(\mathbb{S}^{1}\right)$ с взаимно трансверсальными элементами и групп диффеомор$\oint$ физмов $\mathscr{G} \subseteq \operatorname{Diff}^{1+\alpha}([0,1]), \alpha>0$, нет групп промежуточного роста.

Прокомментируем теорему Наваса (теорема 11). Напомним, что группа Homeo+ $_{+}([0,1])$ гомеоморфизмов интервала $[0,1]$ топологически сопряжена некоторой подгруппе

$$
\eta \circ \mathrm{Homeo}_{+}([0,1]) \circ \eta^{-1}
$$

группы $\mathrm{Homeo}_{+}(\mathbb{R})$, где $\eta:(0,1) \rightarrow \mathbb{R}$ - какой-либо диффеоморфизм класса $C^{\infty}$. Всякой группе $\mathscr{G} \subseteq \operatorname{Diff}^{1+\alpha}([0,1])$ при таком сопряжении соответствует группа

$$
G \subseteq \operatorname{Diff}^{1+\alpha}(\mathbb{R}), \quad \text { где } \quad G=\eta \circ \mathscr{G} \circ \eta^{-1} .
$$

Для группы $\mathscr{G} \subseteq \operatorname{Diff}^{1+\alpha}([0,1])$ определим каноническую нормальную подгруппу

$$
H_{\mathscr{G}}=\eta^{-1} \circ H_{G} \circ \eta,
$$

а также замкнутые инвариантные подмножества

$$
\operatorname{Fix} \mathscr{G}^{S}=\left[\eta^{-1} \circ \operatorname{Fix} G^{S} \circ \eta\right] \cup\{0,1\} \quad \text { и } E(\mathscr{G})=\left[\eta^{-1} \circ E(G) \circ \eta\right] \cup\{0,1\} .
$$

Очевидно, что имеет место канонический изоморфизм групп

$$
G / H_{G} \cong \mathscr{G} / H_{\mathscr{G}} .
$$

Рассмотрим группу $G \subseteq$ Homeo $_{+}(\mathbb{R})$, топологически сопряженную исследуемой группе $\mathscr{G} \subseteq \operatorname{Diff}^{1+\alpha}([0,1])$. Если для группы гомеоморфизмов $G \subseteq$ $\mathrm{Homeo}_{+}(\mathbb{R})$ (а не только для группы диффеоморфизмов) справедливо равенство $H_{G}=\langle e\rangle$ и в ней отсутствует свободная подполугруппа с двумя образующими, то по следствию 6 такая группа является коммутативной; соответственно, коммутативна и сопряженная ей группа $\mathscr{G}$. Остается рассмотреть случай $H_{G} \neq\langle e\rangle$. Для такой группы минимальное множество либо изоморфно канторову множеству, либо является дискретным. В таком случае множество $E(\mathscr{G})$ (минимальное множество) также либо изоморфно канторову множеству на $[0,1]$, либо не может иметь точек накопления, отличных от 0 и 1.

Для группы $\mathscr{G} \subseteq \operatorname{Diff}_{+}^{1+\alpha}([0,1]), \alpha>0$, в силу того, что диффеоморфизмы являются гладкими, включая левый (правый) конец интервала $[0,1]$, удается доказать аналог теоремы Данжуа об отсутствии минимального множества, 
изоморфного канторову множеству. Доказательство основано на том, что максимальные открытые интервалы из дополнения к минимальному множеству не могут накапливаться к точке (в данном случае это левый конец $t=0$ ), в которой диффеоморфизмы имеют гладкость выше единицы.

Остается случай дискретного минимального множества для группы $G$. Из дискретности минимального множества следует, что факторгруппа $G / H_{G}$ не более чем циклическая; соответственно, не более чем циклической является и факторгруппа $\mathscr{G} / H_{\mathscr{G}}$. Рассмотрим дополнение к множеству Fix $\mathscr{G} S$, т. е. множество $[0,1] \backslash \mathrm{Fix}^{\mathscr{C}} \mathscr{G}^{S}$, состоящее из не более чем счетного числа максимальных открытых интервалов $\left\{\left(t_{0 \alpha}, t_{1 \alpha}\right)\right\}_{\alpha \in A}$. Через $H_{\mathscr{G}}(\alpha)$ будем обозначать ограничение действий группы гомеоморфизмов $H_{\mathscr{G}}$ на интервал $\left(t_{0 \alpha}, t_{1 \alpha}\right), \alpha \in A$. Если при этом $H_{\mathscr{g}}=\langle e\rangle$, то все доказано. Остается рассмотреть случай, когда $H_{\mathscr{G}} \neq\langle e\rangle$. В этом случае число нетривиальных групп $H_{G}(\alpha), \alpha \in A$, конечно, иначе соответствующие им интервалы $\left(t_{0 \alpha}, t_{1 \alpha}\right)$ будут накапливаться к некоторой точке, в которой диффеоморфизмы имеют гладкость выше единицы. В частности, отсюда следует, что факторгруппа $\mathscr{G} / H_{\mathscr{G}}$ тривиальна. Остается изучить конечное число нетривиальных групп $H_{\mathscr{G}}(\alpha)$, действующих на интервалах $\left[t_{0 \alpha}, t_{1 \alpha}\right]$. Заметим, что каждая из этих групп обладает всеми исходными свойствами группы $\mathscr{G}$ и, в частности, является конечно порожденной. Поэтому мы можем для каждой из конечного числа нетривиальных групп $H_{G}(\alpha)$ применить всю предыдущую цепочку рассуждений. Такой процесс прерывается через конечное число шагов. Здесь также все основывается на том, что если бы такая процедура не была конечной, то происходило бы накопление интервалов, на которых действуют нетривиальные группы диффеоморфизмов. Но это противоречит условию гладкости больше единицы для диффеоморфизмов в точках накопления. В таком случае через конечное число шагов мы получим, что группа $\mathscr{G}$ является разрешимой. И наконец, применяя теорему Розенблатта (теорема 3 ), получим условие почти нильпотентности группы $\mathscr{G}$.

\section{6. Схема классификации групп гомеоморфизмов прямой (окружности, интервала)}

Проблема классификации групп, в частности групп гомеоморфизмов прямой (окружности, интервала), является одной из центральных задач. Классификация может проводиться с использованием различных характеристик групп. Один из способов классификации - это построение цепи

$$
\langle e\rangle \unlhd \cdots \unlhd G_{i+1} \unlhd G_{i} \unlhd \cdots \unlhd G_{2} \unlhd G_{1} \unlhd G_{0}=G
$$

с заданием единого алгоритма определения нормальной подгруппы $G_{i+1}$ для группы $G_{i}$, при котором факторгруппа $G_{i} / G_{i+1}$ будет принадлежать некоторому фиксированному конечному классу групп. В такой схеме предлагаемая цепь может оказаться и трансфинитной. В связи с этим одной из важных задач является определение групп, для которых такая цепь будет конечной. В конце предыдущего раздела, при обсуждении теоремы Наваса, для групп, не 
содержащих свободных подполугрупп с двумя образующими, была реализована подобная цепь и показана ее конечность.

Приводимая ниже схема классификации групп гомеоморфизмов прямой основана на цепочках вложений факторгрупп, порожденных метрическими инвариантами, которые приводятся в п. 5.1.

Опишем правило формирования цепи для группы $G \subseteq \mathrm{Homeo}_{+}(\mathbb{X})$. Для группы $G=G_{0}$ положим $G_{1}=H_{G_{0}}$.

В случае пустого минимального множества для исходной группы $G_{0}$ (группа принадлежит классу $I \mathscr{W} \backslash I \mathscr{W}(\mathbb{R}))$ по определению полагаем $H_{G_{0}}=\langle e\rangle$. Такая цепь обрывается и имеет ступень, равную единице. Более детальное описание групп с пустым минимальным множеством является отдельной задачей.

Если минимальное множество для исходной группы $G_{0}$ непусто, то по пункту 1 ) предложения 5 факторгруппа $G_{0} / G_{1}$ либо коммутативная (принадлежит классу $I \mathscr{K}(\mathbb{R}))$, либо содержит свободную подполугруппу с двумя образующими (принадлежит классу $\left.I \mathscr{W}(\mathbb{R}) \backslash I \mathscr{N}_{P}(\mathbb{R})\right)$. В случае существования свободной подполугруппы с двумя образующими свойства факторгруппы могут быть уточнены на основании пункта 1$)$ предложения 7 . Если группа $G_{0}$ содержит нормальную подгруппу со свободно действующим элементом и инвариантной мерой, то факторгруппа $G_{0} / G_{1}$ либо является неабелевой разрешимой группой (принадлежит классу $I \mathscr{S}(\mathbb{R}) \backslash I \mathscr{K}(\mathbb{R}))$, либо содержит свободную подгруппу с двумя образующими (принадлежит классу $I \mathscr{W}(\mathbb{R}) \backslash I \mathscr{N}(\mathbb{R}))$. В случае $G_{1}=\langle e\rangle$ цепь обрывается на этом шаге.

Таким образом, факторгруппа $G_{0} / G_{1}$ принадлежит одному из трех классов групп $I \mathscr{W} \backslash I \mathscr{W}(\mathbb{R}), I \mathscr{K}(\mathbb{R}), I \mathscr{W}(\mathbb{R}) \backslash I \mathscr{N}_{P}(\mathbb{R})$. По теореме 27 всякая группа из $I \mathscr{K}(\mathbb{R})$ изоморфна группе сдвигов на прямой. Для групп, имеющих нормальную подгруппу со свободно действующим элементом и инвариантной мерой, последний класс заменяется на два его непересекающихся подмножества $I \mathscr{S}(\mathbb{R}) \backslash I \mathscr{K}(\mathbb{R})$ и $I \mathscr{W}(\mathbb{R}) \backslash I \mathscr{N}(\mathbb{R})$. По теореме 35 всякая группа из $I \mathscr{S}(\mathbb{R}) \backslash I \mathscr{K}(\mathbb{R})$ изоморфна аффинной группе на прямой.

Теперь опишем процедуру формирования нормальной подгруппы $G_{2}$ группы $G_{1}$. Группу $G_{1}$ можно рассматривать как группу гомеоморфизмов, действующих на дополнении к минимальному множеству группы $G_{0}$. Такое дополнение состоит из не более чем счетного числа открытых (максимальных) интервалов, которые обозначим через $\left(t_{0 \alpha}, t_{1 \alpha}\right), \alpha \in \mathscr{A}$. Очевидно, что элементы группы $G_{1}$ на каждом интервале $\left(t_{0 \alpha}, t_{1 \alpha}\right), \alpha \in \mathscr{A}$, действуют как гомеоморфизмы, сохраняющие ориентацию. Выберем произвольное $\alpha \in \mathscr{A}$. Ограничения действий элементов группы $G_{1}$ на интервал $\left(t_{0 \alpha}, t_{1 \alpha}\right), \alpha \in \mathscr{A}$, образуют группу гомеоморфизмов этого интервала. Обозначим такую группу через $G_{1}(\alpha)$. Имеется естественный эпиморфизм $h: G_{1} \rightarrow G_{1}(\alpha)$. Пусть $\eta:\left(t_{0 \alpha}, t_{1 \alpha}\right) \rightarrow \mathbb{R}-$ какой-либо диффеоморфизм класса $C^{\infty}$. Тогда $Q=\eta \circ G_{1}(\alpha) \circ \eta^{-1}$ является группой гомеоморфизмов прямой, сохраняющих ориентацию. Для группы $Q$, как и для группы $G$, мы можем повторить всю процедуру выделения подгруппы $H_{Q}$ и определения факторгруппы $Q / H_{Q}$, которая будет принадлежать одному из классов групп, выделенных выше. Положим $G_{2}=\eta^{-1} h^{-1}\left(G_{1}(\alpha)\right)$. 
Очевидно, что факторгруппа $G_{1} / G_{2}$ изоморфна факторгруппе $Q / H_{Q}$ и принадлежит одному из классов групп, выделенных выше. Такую процедуру можно продолжить по индукции и построить цепь. Неоднозначность выбора последовательности интервалов $\left(t_{0 \alpha}, t_{1 \alpha}\right)$ приводит к неединственности цепи. Изучение свойств таких цепей и позволит провести их классификацию, что эквивалентно классификации групп гомеоморфизмов прямой.

В п. 5.2 рассматривались цепочки вложений для двух типов групп диффеоморфизмов:

$$
\mathscr{G} \subseteq \operatorname{Diff}_{+}^{1+\alpha}([0,1]), \quad \alpha>0, \quad \text { и } \quad \mathscr{G} \subseteq \operatorname{Diff}^{2}\left(\mathbb{S}^{1}\right)
$$

Так как рассматривались цепочки вложений для групп, а не факторгрупп, то это позволило получить дополнительную информацию о факторгруппе $H_{G}$, играющей ключевую роль при формировании цепи. Благодаря этому для таких групп удается установить конечность рассматриваемых цепей.

Для конечно порожденных групп диффеоморфизмов $\mathscr{G} \subseteq \operatorname{Diff}_{+}^{1+\alpha}([0,1])$, $\alpha>0$, предлагаемая цепь имеет простую и обозримую структуру. При обсуждении теоремы Наваса мы отмечали, что для таких групп отсутствуют минимальные множества, изоморфные канторову множеству. Выше мы уже отмечали, что на каждой ступени цепи, в случае минимального множества, совпадающего со всем пространством действия, цепь обрывается. Учитывая это и повторяя пункты обсуждения теоремы Наваса, получим, что такая цепь конечна.

Для конечно порожденных групп диффеоморфизмов $G \subseteq \operatorname{Diff}^{2}\left(\mathbb{S}^{1}\right)$ с взаимно трансверсальными элементами предлагаемая цепь также имеет простую и обозримую структуру. Мы уже отмечали, что для таких групп также отсутствуют минимальные множества, изоморфные канторову множеству. Доказательство этого факта приводится в работе [16]. Там же показано, что для таких групп представленная цепь имеет ступень не более двух. В случае ступени один группа либо имеет свободную подгруппу с двумя образующими, либо является коммутативной. В случае ступени два факторгруппа $G / G_{1}$ циклическая, а нормальная подгруппа $G_{1}$, как и должно быть, коммутативная.

Приводимые примеры призваны продемонстрировать возможности представленной цепи в вопросах классификации групп гомеоморфизмов прямой (окружности, интервала).

Автор признателен всем участникам семинара "Динамические системы и эргодическая теория" и его руководителям Д. В. Аносову и А. М. Стёпину за полезные советы и внимание к работе.

\section{Список литературы}

[1] С. И. Адян, Проблема Бернсайда и тождества в группах, Наука, М., 1975, 335 с.; англ. пер.: S. I. Adian, The Burnside problem and identities in groups, Ergeb. Math. Grenzgeb., 95, Springer-Verlag, Berlin-New York, 1979, xi+311 pp.

[2] С. И. Адян, "Случайные блуждания на свободных периодических группах", Изв. АН СССР. Сер. матем., 46:6 (1982), 1139-1149; англ. пер.: S. I. Adian, "Random walks on free periodic groups", Math. USSR-Izv., 21:3 (1983), 425-434. 
[3] Л. Альфорс, Лекиии по квазиконформным отображениям, Мир, М., 1969, 133 с.; пер. с англ.: L.V. Ahlfors, Lectures on quasiconformal mappings, Van Nostrand Mathematical Studies, 10, D. Van Nostrand Co., Inc., Toronto, ON-New YorkLondon, 1966, v+146 pp.

[4] Л. Альфорс, Преобразования Мёбиуса в многомерном пространстве, Мир, М., 1986, 112 с.; пер. с англ.: L. V. Ahlfors, Möbius transformations in several dimensions, Ordway Professorship Lectures Math., Univ. Minnesota, School of Mathematics, Minneapolis, MN, 1981, ii+150 pp.

[5] Д.В. Аносов, "О вкладе Н.Н. Боголюбова в теорию динамических систем", УМH, 49:5(299) (1994), 5-20; англ. пер.: D. V. Anosov, "On the contribution of N. N. Bogolyubov to the theory of dynamical systems", Russian Math. Surveys, 49:5 (1994), 1-18.

[6] Л. А. Бекларян, "Структура факторгруппы группы гомеоморфизмов $\mathbb{R}$, сохраняющих ориентацию, по подгруппе, порожденной объединением стабилизаторов", Докл. РАН, 331:2 (1993), 137-139; англ. пер.: L. A. Beklaryan, "The structure of the quotient group of the group of orientation-preserving homeomorphisms of $\mathbb{R}$ by the subgroup generated by the union of the stabilizers", Russian Acad. Sci. Dokl. Math., 48:1 (1994), 37-39.

[7] Л. А. Бекларян, "Инвариантные и проективно инвариантные меры для групп гомеоморфизмов $\mathbb{R}$ ", Докл. РАH, 332:6 (1993), 679-681; англ. пер.: L. A. Beklaryan, "Invariant and projectively invariant measures for groups of orientation-preserving homeomorphisms of $\mathbb{R}^{\prime}$, Russian Acad. Sci. Dokl. Math., 48:2 (1994), 387-390.

[8] Л.А. Бекларян, "K вопросу о классификации групп гомеоморфизмов $\mathbb{R}$, сохраняющих ориентацию. І. Инвариантные меры", Матем. сб., 187:3 (1996), 23-54; англ. пер.: L. A. Beklaryan, "On the classification of groups of orientation-preserving homeomorphisms of $\mathbb{R}$. I. Invariant measures", Sb. Math., 187:3 (1996), 335-364.

[9] Л. А. Бекларян, "Критерий существования проективно инвариантной меры для групп гомеоморфизмов $\mathbb{R}$, сохраняющих ориентацию, связанный со структурой множества неподвижных точек", УМH, 51:3(309) (1996), 179-180; англ. пер.: L. A. Beklaryan, "A criterion connected with the structure of the fixed-point set for the existence of a projectively invariant measure for groups of orientation-preserving homeomorphisms of $\mathbb{R}^{\prime \prime}$, Russian Math. Surveys, 51:3 (1996), 539-540.

[10] Л. А. Бекларян, "K вопросу о классификации групп гомеоморфизмов $\mathbb{R}$, сохраняющих ориентацию. II. Проективно-инвариантные меры", Матем. сб., 187:4 (1996), 3-28; англ. пер.: L. A. Beklaryan, "On the classification of groups of orientation-preserving homeomorphisms of $\mathbb{R}$. II. Projectively-invariant measures", Sb. Math., 187:4 (1996), 469-494.

[11] Л.А. Бекларян, "K вопросу о классификации групп гомеоморфизмов $\mathbb{R}$, сохраняющих ориентацию. III. $\omega$-проективно-инвариантные меры”, Матем. сб., 190:4 (1999), 43-62; англ. пер.: L. A. Beklaryan, "On the classification of groups of orientation-preserving homeomorphisms of $\mathbb{R}$. III. $\omega$-projectively invariant measures", Sb. Math., 190:4 (1999), 521-538.

[12] Л.А. Бекларян, "О критерии топологической сопряженности квазисимметрической группы группе аффинных преобразований $\mathbb{R} "$, Матем. сб., 191:6 (2000), 31-42; англ. пер.: L. A. Beklaryan, "On a criterion for the topological conjugacy of a quasisymmetric group to a group of affine transformations of $\mathbb{R}^{\prime \prime}$, Sb. Math., 191:6 (2000), 809-819.

[13] Л.А. Бекларян, "Об аналогах альтернативы Титса для групп гомеоморфизмов окружности и прямой", Матем. заметки, 71:3 (2002), 334-347; англ. пер.: L. A. Beklaryan, "On analogs of the Tits alternative for groups of homeomorphisms of the circle and of the line", Math. Notes, 71:3 (2002), 305-315. 
[14] Л.А. Бекларян, "Группы гомеоморфизмов прямой и окружности. Топологические характеристики и метрические инварианты", УМН, 59:4(358) (2004), 3-68; англ. пер.: L. A. Beklaryan, "Groups of homeomorphisms of the line and the circle. Topological characteristics and metric invariants", Russian Math. Surveys, 59:4 (2004), 599-660.

[15] Л.А. Бекларян, Введение в теорию функиионально-дифференциалъных уравнений. Групповой подход, Факториал Пресс, М., 2007, 288 с.

[16] L.A. Beklaryan, "Group specialities in the problem of the maximum principle for systems with deviating argument", J. Dyn. Control Syst., 18:3 (2012), 419-432.

[17] Л.А. Бекларян, "О массивных подмножествах в пространстве конечно-порожденных групп диффеоморфизмов окружности", Матем. заметки, 92:6 (2012), 825-833; англ. пер.: L. A. Beklaryan, "Residual subsets in the space of finitely generated groups of diffeomorphisms of the circle", Math. Notes, 93:1 (2013), 29-35.

[18] Л.А. Бекларян, "Критерии существования инвариантной меры для групп гомеоморфизмов прямой", Матем. заметки, 95:3 (2014), 335-339; англ. пер.: L.A. Beklaryan, "Criteria for the existence of an invariant measure for groups of homeomorphisms of the line", Math. Notes, 95:3 (2014), 304-307.

[19] Л. А. Бекларян, "Группы гомеоморфизмов прямой. Критерии существования инвариантной и проективно инвариантной мер в терминах коммутанта", Матем. сб., 205:12 (2014), 63-84; англ. пер.: L. A. Beklaryan, "Groups of homeomorphisms of the line. Criteria for the existence of invariant and projectively invariant measures in terms of the commutator subgroup", Sb. Math., 205:12 (2014), 1741-1760.

[20] M. C. Brin, C.C. Squier, "Groups of piecewice linear homeomorphisms on the real line", Invent. Math., 79:3 (1985), 485-498.

[21] J. F. Cannon, W. J. Floyd, W. R. Parry, "Introductory notes on Richard Thompson's groups", Enseign. Math. (2), 42:3-4 (1996), 215-256; PЖMam, 1998, 1L203.

[22] Т. Г. Чеккерини-Зилберштейн, "Вокруг аменабельности", Труды международной конферениии, посвященной 90-летию со дня рождения Л. С. Понтрягина (Москва, 31 августа-6 сентября 1998 г.). Том 8. Алгебра, Итоги науки и техн. Сер. Соврем. матем. и ее прил. Темат. обз., 69, ВИНИТИ, М., 1999, 229-259; англ. пер.: T. G. Ceccherini-Silberstein, "Around amenability", J. Math. Sci. (N. Y.), 106:4 (2001), 3145-3163.

[23] M. M. Day, "Amenable semigroups", Illinois J. Math., 1:4 (1957), 509-544.

[24] П. де ля Арп, Р.И. Григорчук, Т. Чекерини-Сильберстайн, “Аменабельность и парадоксальные разбиения для псевдогрупп и дискретных метрических пространств", Алгебра. Топология. Дифференииальные уравнения и их приложения, Сборник статей. К 90-летию со дня рождения академика Льва Семеновича Понтрягина, Тр. МИАН, 224, Наука, М., 1999, 68-111; англ. пер.: R. I. Grigorchuk, T. Ceccherini-Silberstein, P. de la Harpe, "Amenability and paradoxical decompositions for pseudogroups and for discrete metric spaces", Proc. Steklov Inst. Math., 224 (1999), 57-97.

[25] B. Deroin, V. Kleptsyn, A. Navas, "Sur la dynamique unidimensionnelle en régularité intermédiaire", Acta Math., 199:2 (2007), 199-262.

[26] M. Ershov, G. Golan, M. Sapir, The Tarski numbers of groups, 2014, 26 pp., arXiv: 1401.2202.

[27] B. Farb, J. Franks, "Groups of homeomorphisms of one-manifolds. III. Nilpotent supgroups", Ergodic Theory Dynam. Systems, 23:5 (2003), 1467-1484.

[28] É. Ghys, "Groups acting on the circle", Enseign. Math. (2), 47:3-4 (2001), 329-407.

[29] Ф. Гринлиф, Инвариантные средние на топологических группах и их приложения, Мир, М., 1973, 136 с.; пер. с англ.: F. P. Greenleaf, Invariant means on topological groups and their applications, Van Nostrand Mathematical Studies, 16, Van Nostrand Reinhold Co., New York-Toronto, ON-London, 1969, ix+113 pp. 
[30] Р. И. Григорчук, "Степени роста конечно-порожденных групп и теория инвариантных средних", Изв. АН СCCP, 48:5 (1984), 939-985; англ. пер.: R. I. Grigorchuk, "Degrees of growth of finitely generated groups, and the theory of invariant means", Math. USSR-Izv., 25:2 (1985), 259-300.

[31] Р. И. Григорчук, "Пример конечно определенной аменабельной группы, не принадлежащей классу EG", Матем. сб., 189:1 (1998), 79-100; англ. пер.: R. I. Grigorchuk, "An example of a finitely presented amenable group not belonging to the class EG", Sb. Math., 189:1 (1998), 75-95.

[32] Р. И. Григорчук, П.Ф. Курчанов, "Некоторые вопросы теории групп, связанные с геометрией", Алгебра - 7, Итоги науки и техн. Сер. Соврем. пробл. матем. Фундам. направления, 58, ВИНИТИ, М., 1990, 191-256; англ. пер.: R. I. Grigorchuk, P. F. Kurchanov, "Some questions of group theory related to geometry", Algebra, VII, Encyclopaedia Math. Sci., 58, Springer, Berlin, 1993, 167-232.

[33] Р.И. Григорчук, А. Маки, "О группе промежуточного роста, действующей на прямой гомеоморфизмами", Матем. заметки, 53:2 (1993), 46-63; англ. пер.: R. I. Grigorchuk, A. Maki, "A group of intermediate growth acting by homomorphisms on the real line", Math. Notes, 53:2 (1993), 146-157.

[34] M. Gromov, "Hyperbolic groups", Essays in group theory, Math. Sci. Res. Inst. Publ., 8, Springer, New York, 1987, 75-263.

[35] M. Gromov, "Groups of polynomial growth and expanding maps", Inst. Hautes Études Sci. Publ. Math., 53:1 (1981), 53-78.

[36] Ю. И. Карлович, "C ${ }^{*}$-алгебра операторов типа свертки с дискретными группами сдвигов и осциллирующими коэффициентами", Докл. АН СCCP, 302:3 (1988), 535-540; англ. пер.: Yu. I. Karlovich, " $C^{*}$-algebras of operators of convolution type with discrete groups of shifts and with oscillating coefficients", Soviet Math. Dokl., 38:2 (1989), 301-307.

[37] М. М. Крылов, М. М. Боголюбов, “Загальна теорія міри та іi застосувания до вівчения динамичніх систем нелінійній механіці”, Збірник прачь з нелінійной механіки. Записки кафедри математичноі фізики Інституту будівельноі механіки АН УРСР, 3 (1937), 55-112; фр. изд.: N. Kryloff, N. Bogoliouboff, "La théorie générale de la mesure dans son application à l'étude des systèmes dynamiques de la mécanique non linéaire", Ann. of Math. (2), 38:1 (1937), 65-113.

[38] И.П. Корнфельд, Я.Г. Синай, С.В. Фомин, Эргодическая теория, Наука, М., 1980, 384 с.; англ. пер.: I. P. Cornfeld, S. V. Fomin, Ya. G. Sinai, Ergodic theory, Grundlehren Math. Wiss., 245, Springer-Verlag, New York, 1982, x+486 pp.

[39] А. Г. Курош, Лекиии по общей алгебре, 2-е изд., Наука, М., 1973, 399 с.; англ. пер. 1-го изд.: А. G. Kurosh, Lectures in general algebra, Internat. Ser. Monogr. Pure Appl. Math., 70, Pergamon Press, Oxford-Edinburgh-New York, 1965, x+364 pp.

[40] G. Margulis, "Free subgroups of the homeomorphism group of the circle", C. R. Acad. Sci. Paris Sér. I Math., 331:9 (2000), 669-674.

[41] A. Navas, "Group of circle diffeomorphisms", 2006 (v3 - 2009), 224, arXiv: math/0607481.

[42] С. П. Новиков, "Топология слоений”, Тр. ММО, 14, Изд-во Моск. ун-та, М., 1965, 248-278; англ. пер.: S. P. Novikov, "Topology of foliations", Trans. Moscow Math. Soc., 14, Amer. Math. Soc., Providence, RI, 1965, 268-304.

[43] А.Ю. Ольшанский, "K вопросу о существовании инвариантного среднего на группе", УМH, 35:4(214) (1980), 199-200; англ. пер.: A. Yu. Ol'shanskii, "On the problem of the existence of an invariant mean on a group", Russian Math. Surveys, 35:4 (1980), 180-181.

[44] J. F. Plante, "Foliations with measure preserving holonomy", Ann. of Math. (2), 102:2 (1975), 327-361. 
[45] J.F. Plante, "Solvable groups acting on the line", Trans. Amer. Math. Soc., 278:1 (1983), 401-414.

[46] J.F. Plante, W.P. Thurston, "Polynomial growth in holonomy groups of foliations", Comment. Math. Helv., 51:4 (1976), 567-584.

[47] А. Робертсон, В. Робертсон, Топологические векторные пространства, Мир, М., 1967, 257 с.; пер. с англ.: А. P. Robertson, W. J. Robertson, Topological vector spaces, Cambridge Tracts in Mathematics and Mathematical Physics, 53, Cambridge Univ. Press, New York, 1964, viii+158 pp.

[48] J. M. Rosenblatt, "Invariant measures and growth conditions", Trans. Amer. Math. Soc., 193 (1974), 33-53.

[49] E. Salhi, "Sur les ensembles minimaux locaux", C. R. Acad. Sci. Paris Sér. I Math., 295:12 (1982), 691-694.

[50] E. Salhi, "Sur un théorème de structure de feuilletages de codimension 1", C. R. Acad. Sci. Paris Sér. I Math., 300:18 (1985), 635-638.

[51] E. Salhi, "Niveau de feuilles", C. R. Acad. Sci. Paris Sér. I Math., 301:5 (1985), 219-222.

[52] M. V. Sapir, Combinatorial algebra: syntax and semantics, Springer Monogr. Math., Springer, Cham, 2014, xvi+355 pp.

[53] В.В.Солодов, "Гомеоморфизмы прямой и слоения", Изв. АН СССР. Сер. матем., 46:5 (1982), 1047-1060; англ. пер.: V. V. Solodov, "Homeomorphisms of the line and a foliation", Math. USSR-Izv., 21:2 (1983), 341-354.

[54] В.В. Солодов, "Гомеоморфизмы окружности и слоения", Изв. АН СССР. Сер. матем., 48:3 (1984), 599-613; англ. пер.: V. V. Solodov, "Homeomorphisms of the circle and a foliation", Math. USSR-Izv., 24:3 (1985), 553-566.

[55] А.М. Степин, "Аппроксимируемость групп и групповых действий”, УМН, 38:6(234) (1983), 123-124; англ. пер.: A. M. Stepin, "Approximability of groups and group actions", Russian Math. Surveys, 38:6 (1983), 131-132.

[56] A. M. Stepin, "Approximation of groups and group actions, the Cayley topology", Ergodic theory of $\mathbb{Z}^{d}$ actions (Warwick, 1993-1994), London Math. Soc. Lecture Note Ser., 228, Cambridge Univ. Press, Cambridge, 1996, 475-484.

[57] A. Tarski, Cardinal algebras, Oxford Univ. Press, New York, NY, 1949, xii+326 pp.

[58] Ж. Титс, "Свободные подгруппы линейных групп", Математика. Сб. пер., 16, № 2, Мир, М., 1972, 47-66; пер. с англ.: J. Tits, "Free subgroups in linear groups", J. Algebra, 20:2 (1972), 250-270.

[59] А.М. Вершик, "Счетные группы, близкие к конечным", Добавление к кн.: Ф. Гринлиф, Инвариантные средние на топологических группах и их приложения, Мир, М., 1973, 112-135.

[60] S. Wagon, The Banach-Tarski paradox, Encyclopedia Math. Appl., 24, Camdridge Univ. Press, Cambridge, 1985, xvi+251 pp.

Лева Андреевич Бекларян

Поступила в редакцию

(Leva A. Beklaryan)

07.05 .2014

Центральный экономико-математический

институт РАН

E-mail: beklar@cemi.rssi.ru 